\title{
Non-variational computation of the eigenstates of Dirac operators with radially symmetric potentials
}

\author{
Lyonell Boulton and Nabile Boussaid
}

\begin{abstract}
We discuss a novel strategy for computing the eigenvalues and eigenfunctions of the relativistic Dirac operator with a radially symmetric potential. The virtues of this strategy lie in the fact that it avoids completely the phenomenon of spectral pollution and it always provides two-sided estimates for the eigenvalues with explicit error bounds on both eigenvalues and eigenfunctions. We also discuss convergence rates of the method and illustrate our results with various numerical experiments.
\end{abstract}

\section{Introduction}

The free Dirac operator acting on 4 -spinors of $L^{2}\left(\mathbb{R}^{3}\right)^{4}$ is determined by the first-order differential expression

$$
D:=\alpha \cdot P+\beta=-\mathrm{i} \sum_{k=1}^{3} \alpha_{k} \partial_{k}+\beta,
$$

where $\alpha=\left(\alpha_{1}, \alpha_{2}, \alpha_{3}\right)$, and the Pauli-Dirac matrices are

$$
\begin{gathered}
\alpha_{i}=\left(\begin{array}{cc}
0 & \sigma_{i} \\
\sigma_{i} & 0
\end{array}\right) \quad \text { and } \beta=\left(\begin{array}{cc}
I_{\mathbb{C}^{2}} & 0 \\
0 & -I_{\mathbb{C}^{2}}
\end{array}\right), \\
\text { for } \sigma_{1}=\left(\begin{array}{ll}
0 & 1 \\
1 & 0
\end{array}\right), \quad \sigma_{2}=\left(\begin{array}{cc}
0 & -\mathrm{i} \\
\mathrm{i} & 0
\end{array}\right) \quad \text { and } \quad \sigma_{3}=\left(\begin{array}{cc}
1 & 0 \\
0 & -1
\end{array}\right) .
\end{gathered}
$$

We assume that the units are fixed so that $m=c=\hbar=1$. Standard arguments involving the Fourier transform show that $D$ defines a self-adjoint operator with domain $H^{1}\left(\mathbb{R}^{3}\right)^{4}$ and that the spectrum of $D$ is

$$
\operatorname{Spec}(D)=(-\infty,-1] \cup[1, \infty)
$$

Spherically symmetric potentials are Hermitian $4 \times 4$ matrix multiplication operators, $V$, acting on $L^{2}\left(\mathbb{R}^{2}\right)^{4}$, such that $\mathcal{C}_{0}^{\infty}\left(\mathbb{R}^{3} \backslash\{0\}\right) \subset \operatorname{Dom}(V)$ and

$$
e^{\mathrm{i} \varphi n \cdot S} V\left(R^{-1} x\right) e^{-\mathrm{i} \varphi n \cdot S}=V(x), \quad \forall x \in \mathbb{R}^{3}, \forall \varphi \in[0,4 \pi),
$$

where

$$
S=\frac{1}{2}\left(\begin{array}{ll}
\sigma & 0 \\
0 & \sigma
\end{array}\right)
$$

is the spin operator, and $R$ is the matrix of the rotation of angle $\varphi$ and axis $n$. Here $\operatorname{Dom}(V)$ denotes the maximal domain of $V$.

Spherically symmetric potentials may be constructed from maps

$$
\phi_{\mathrm{sc}, \mathrm{el}, \mathrm{am}}: \mathbb{R} \longrightarrow \mathbb{R}
$$

Received 20 November 2008; revised 28 March 2009.

2000 Mathematics Subject Classification 47B36 (primary), 47B39, 81-08 (secondary). 
via

$$
V(x)=\phi_{\mathrm{sc}}(|x|) \beta+\phi_{\mathrm{el}}(|x|) I_{\mathbb{C}^{4}}+i \phi_{\mathrm{am}}(|x|) \beta \alpha \cdot \frac{x}{|x|} .
$$

The subscripts 'sc', 'el' and 'am' stand for 'scalar', 'electric' and 'anomalous magnetic' potential, respectively. Radial symmetry on the electric potential, for instance, is a consequence of the assumption that the atomic nucleus is pointwise and the electric forces are isotropic in an isotropic medium like a vacuum. In the particular case $\phi_{\mathrm{sc}}=\phi_{\mathrm{am}}=0$ and $\phi_{\mathrm{el}}(r)=\gamma / r$, where $\sqrt{3} / 2<\gamma<0$, we see that $D+V$ describes the motion of a relativistic electron in the field created by an atomic nucleus.

If $V$ is subject to suitable smallness and regularity conditions (such as those ensuring that $V$ is relatively compact with respect to $D$ ), then $H:=D+V$ defines an essentially self-adjoint operator in $C_{0}^{\infty}\left(\mathbb{R}^{3} \backslash\{0\}\right)^{4}$ with self-adjoint extension domain $H^{1}\left(\mathbb{R}^{3}\right)^{4}$ and

$$
\operatorname{Spec}_{\text {ess }}(H)=\operatorname{Spec}(D)=(-\infty,-1] \cup[1, \infty),
$$

see [19, Theorems 4.2 and 4.16]. In fact there are known conditions, satisfied by potentials of practical interest (see, for example, [1, Theorems 6 and A]), preventing the existence of embedded eigenvalues.

The addition of a non-zero potential might give rise to a non-empty discrete spectrum in the gap $(-1,1)$. These eigenvalues can only be found explicitly for a few simple systems which do not go much beyond the case of a single electron embedded in a field created by an atomic nucleus; see (4.1). For more complicated potentials, one has to rely either on asymptotic techniques (cf. $[4,10,15]$ and references therein) or on numerical estimations.

Few robust computational procedures are currently available to estimate numerically the eigenvalues of $H$; see $[\mathbf{8}, \mathbf{9}, \mathbf{1 3}]$ and the references therein. Since $H$ is strongly indefinite, a direct implementation of the Galerkin method is not possible due to variational collapse. Under no further restriction on the potential or the reduction basis, accumulation points of eigenvalues of the finite-dimensional approximate operator which do not belong to $\operatorname{Spec}(H)$ might appear; see [17] and [8]. This phenomenon is known as spectral pollution.

The aim of the present paper is to investigate the applicability of the so-called quadratic projection method for finding eigenvalues and eigenfunctions of $H$. This method has been recently studied in an abstract setting (see $[\mathbf{5}, \mathbf{1 4}, \mathbf{1 6}]$ and $[\mathbf{6}]$ ) and it has already been applied with some success to crystalline Schrödinger operators [7] and magnetohydrodynamics [18]. In this approach, explained at length in Section 3, the underlying discretised eigenvalue problem is quadratic in the spectral parameter (rather than linear) and has non-real eigenvalues. Its main advantage over a standard Galerkin method is its robustness: it never pollutes and it always provides a posteriori two-sided estimates of the error of computed eigenvalues.

Section 3.1 is devoted to a self-contained description of the quadratic projection method. In Theorem 1 we present an alternative proof of Shargorodsky's non-pollution Theorem [14, Theorem 2.6]. In addition, we show that information about eigenfunctions can be recovered from the quadratic projection method, see (3.4).

In Section 4 we test the practical applicability of the numerical scheme proposed in Section 3 by reporting on various numerical experiments. As benchmark potentials we have chosen the purely coulombic, sub-coulombic and inverse harmonic electric potentials. In order to perform these numerical experiments, we split the space into upper and lower spinor components, after writing the problem in radial form. Spectral pollution in the standard Galerkin method using this decomposition and the consequences of unbalancing the number of upper/lower components is discussed in Section 2.

We have chosen a basis of Hermite functions to reduce the continuous problem into finitedimensional from. In Section 3.3 we perform a rigorous convergence analysis of the quadratic method using this basis. This analysis relies upon the general result [6, Theorem 2.1]. The surprising numerical evidence included in Section 4.4 strongly suggests that, under 
appropriate circumstances, choosing a basis that heavily pollutes the standard Galerkin method significantly improves convergence rates of the quadratic projection method.

We have posted a fully functional Matlab code for assembling the matrices involved in the calculations performed in this paper, for $V$ a coulombic potential, in the Manchester NLEVP collection of non-linear eigenvalue problems [3]. See the permanent link [2]. In the Appendix we include details of the explicit expressions of all matrix coefficients.

\section{Spectral pollution and upper/lower spinor component balance}

Consider, for any $\Psi \in L^{2}\left(\mathbb{R}^{3}\right)^{4}$, the spherical coordinates representation:

$$
\psi(r, \theta, \phi)=r \Psi(r \sin (\theta) \sin (\phi), r \sin (\theta) \cos (\phi), r \cos (\theta))
$$

where $(r, \theta, \phi) \in(0, \infty) \times[0, \pi) \times[-\pi, \pi)$. The map $\Psi \mapsto \psi$ is an isomorphism between $L^{2}\left(\mathbb{R}^{3}\right)^{4}$ and $L^{2}((0, \infty), d r) \otimes L^{2}\left(S^{2}\right)^{4}$. If we decompose $L^{2}\left(S^{2}\right)^{4}$ as the sum of the so-called twodimensional angular momentum subspaces $\mathfrak{K}_{m_{j}, \kappa_{j}}$ (see $[\mathbf{1 9}$, Section 4.6]), partial wave subspaces are given by

$$
\mathfrak{H}_{m_{j}, \kappa_{j}}=L^{2}((0, \infty), d r) \otimes \mathfrak{K}_{m_{j}, \kappa_{j}},
$$

so that $L^{2}\left(\mathbb{R}^{3}\right)^{4}=\oplus \mathfrak{H}_{m_{j}, \kappa_{j}}$. Without further mention, here and below we always assume that the indices $\left(m_{j}, \kappa_{j}\right)$ run over the set $m_{j} \in\{-j, \ldots, j\}$ and $\kappa_{j} \in\left\{ \pm\left(j+\frac{1}{2}\right)\right\}$, for $j \in$ $\{(2 k+1) / 2: k \in \mathbb{N}\}$.

The $r$ factor in (2.1) renders a Dirichlet boundary condition at 0 . The dense subspaces $C_{0}^{\infty}(0, \infty) \otimes \mathfrak{K}_{m_{j}, \kappa_{j}} \subset \mathfrak{H}_{m_{j}, \kappa_{j}}$ are invariant under the action of $H$. If $V$ is as in (1.1), then $H \uparrow C_{0}^{\infty}(0, \infty) \otimes \mathfrak{K}_{m_{j}, \kappa_{j}}$ is unitary equivalent to

$$
H_{m_{j}, \kappa_{j}}:=\left(\begin{array}{cc}
1+\phi_{\mathrm{sc}}(r)+\phi_{\mathrm{el}}(r) & -\frac{d}{d r}+\frac{\kappa_{j}}{r}+\phi_{\mathrm{am}}(r) \\
\frac{d}{d r}+\frac{\kappa_{j}}{r}+\phi_{\mathrm{am}}(r) & -1-\phi_{\mathrm{sc}}(r)+\phi_{\mathrm{el}}(r)
\end{array}\right) .
$$

The operators $H_{m_{j}, \kappa_{j}}$ are essentially self-adjoint in $C_{0}^{\infty}(0, \infty)^{2}$ under suitable conditions on the potentials $\phi_{\mathrm{sc}, \mathrm{el}, \mathrm{am}}$. Then

$$
\operatorname{Spec}(H)=\overline{\bigcup \operatorname{Spec}\left(H_{m_{j}, \kappa_{j}}\right)} .
$$

Below we often suppress the sub-index $\left(m_{j}, \kappa_{j}\right)$ from operators and spaces, and only write the index $\kappa \equiv \kappa_{j}$. Note that the eigenvalues of $H$ are degenerate and their multiplicity is at least $m_{j}$. By virtue of (1.2),

$$
\operatorname{Spec}_{\text {disc }}(H)=\bigcup \operatorname{Spec}_{\text {disc }}\left(H_{\kappa}\right) .
$$

Since $\operatorname{Spec}_{\text {ess }}\left(H_{\kappa}\right)=(-\infty,-1] \cup[1, \infty)$, the $H_{\kappa}$ are strongly indefinite.

Let us consider a heuristic approach to the problem of spectral pollution for $H_{\kappa}$ and the decomposition of $L^{2}(0, \infty)^{2}$ into upper and lower spinor components. For simplicity, we assume that the potential is purely electric and attractive, $\phi_{\mathrm{sc}}(r)=\phi_{\mathrm{am}}(r)=0$ and $\phi_{\mathrm{el}}(r)<0$.

The pair $(u, v) \in \operatorname{Dom}\left(H_{\kappa}\right)$ is a wave function of $H_{\kappa}$ with associated eigenvalue $E \in(-1,1)$ if and only if

$$
\left(\phi_{\mathrm{el}}+1-E\right) u+\left(-\partial_{r}+\frac{\kappa}{r}\right) v=0 \quad \text { and } \quad\left(\partial_{r}+\frac{\kappa}{r}\right) u+\left(\phi_{\mathrm{el}}-1-E\right) v=0 .
$$

The system (2.3) can be easily decoupled into

$$
L_{E} u=0 \quad \text { and } \quad v=-\left(\phi_{\mathrm{el}}-1-E\right)^{-1}\left(\partial_{r}+\frac{\kappa}{r}\right) u,
$$


where

$$
\begin{aligned}
L_{E} & :=-\left(-\partial_{r}+\frac{\kappa}{r}\right)\left(\phi_{\mathrm{el}}-1-E\right)^{-1}\left(\partial_{r}+\frac{\kappa}{r}\right)+\left(\phi_{\mathrm{el}}+1-E\right) \\
& \geqslant(1+E)^{-1}\left(\partial_{r}+\frac{\kappa}{r}\right)^{*}\left(\partial_{r}+\frac{\kappa}{r}\right)+\phi_{\mathrm{el}}+(1-E) .
\end{aligned}
$$

If we assume $\phi_{\mathrm{el}}$ is relatively compact with respect to the non-negative operator $\left(\partial_{r}+\right.$ $(\kappa / r))^{*}\left(\partial_{r}+(\kappa / r)\right)$, then

$$
\min \operatorname{Spec}_{\mathrm{ess}} L_{E} \geqslant(1-E)>0 .
$$

Moreover, the expression for $v$ in (2.4) yields $u \not \equiv 0$ and $v \not \equiv 0$. Hence $E \in \operatorname{Spec}_{\text {disc }} H_{\kappa}$ if and only if 0 is in the discrete spectrum of $L_{E}$.

Let $\mathcal{M}_{N} \subset L^{2}(0, \infty)$ be a nested family of finite-dimensional subspaces such that $\mathcal{M}_{N} \subset$ $\mathcal{M}_{N+1}$,

$$
\overline{\bigcup_{N \geqslant 1} \mathcal{M}_{N}}=L^{2}(0, \infty)
$$

and $\mathcal{L}_{N M}:=\mathcal{M}_{N} \oplus \mathcal{M}_{M} \subset \operatorname{Dom}\left(H_{\kappa}\right)$. Let $P_{N}$ denote the orthogonal projection onto $\mathcal{M}_{N}$, so that $P_{N} \rightarrow I$ in the strong sense. With this notation we wish to apply the Galerkin method to the operator $H_{\kappa}$ with test spaces $\mathcal{L}_{N M}$.

We first consider the case $N=M$. Let $\left(u_{N}, v_{N}\right)^{t} \in \mathcal{L}_{N N}$ be a sequence normalised by $\left\|u_{N}\right\|^{2}+\left\|v_{N}\right\|^{2}=1$, such that

$$
\begin{gathered}
P_{N}\left[\left(\phi_{\mathrm{el}}+1-E_{N}\right) u_{N}+\left(-\partial_{r}+\frac{\kappa}{r}\right) v_{N}\right]=0, \\
P_{N}\left[\left(\partial_{r}+\frac{\kappa}{r}\right) u_{N}+\left(\phi_{\mathrm{el}}-1-E_{N}\right) v_{N}\right]=0
\end{gathered}
$$

for a suitable sequence $E_{N} \rightarrow \tilde{E} \in(-1,1)$. Let $\chi_{N}$ be such that

$$
v_{N}=-\left(\phi_{\mathrm{el}}-1-E_{N}\right)^{-1}\left(\partial_{r}+\frac{\kappa}{r}\right) u_{N}+\chi_{N} .
$$

By virtue of (2.7),

$$
P_{N}\left(\phi_{\mathrm{el}}-1-E_{N}\right) \chi_{N}=0 .
$$

If we were able to prove that

$$
\left\|P_{N}\left(-\partial_{r}+\frac{\kappa}{r}\right) \chi_{N}\right\| \rightarrow 0, \quad N \rightarrow \infty,
$$

by substituting into (2.6), we would have $\left\|P_{N} L_{\tilde{E}} u_{N}\right\| \rightarrow 0$. Thus, by virtue of the min-max principle alongside with (2.5), we would have $0 \in \operatorname{Spec}_{\text {disc }} L_{\tilde{E}}$ and so $\tilde{E} \in \operatorname{Spec}_{\text {disc }} H_{\kappa}$.

Suppose now that $M: \mathbb{N} \longrightarrow \mathbb{N}$. Let $\left(u_{N}, v_{N}\right)^{t} \in \mathcal{L}_{N M(N)}$ be a sequence normalised by $\left\|u_{N}\right\|^{2}+\left\|v_{N}\right\|^{2}=1$, such that (2.6) holds true and $P_{N}$ is replaced in (2.7) by $P_{M(N)}$. If $\lim _{N \rightarrow \infty} M(N) / N<1$, we are certainly less likely to obtain (2.9) as $P_{N}$ is replaced in (2.8) by $P_{M(N)}$. If, on the other hand, $\lim _{N \rightarrow \infty} M(N) / N>1$, then we would be more confident about obtaining (2.9). In Section 4.4 we will present a series of numerical experiments supporting the validity of this argumentation. In particular, spectral pollution in the standard Galerkin method appears to increase as $\lim _{N \rightarrow \infty} M(N) / N$ decreases (see Figure 4).

In practice, (2.9) is difficult to verify for particular choices of $\mathcal{M}_{N}$. Nevertheless, however, the above heuristics is the basis of rigorous pollution-free numerical procedures for computing eigenvalues of $H_{\kappa}$. One such procedure is that developed by Dolbeault, Esteban and Séré (cf. the recent review [9]). Multiplying by $u$ the left-hand equation of (2.4) and integrating in 
the space variable gives $A(E) u=0$ for

$$
A(\lambda) v:=\int_{0}^{\infty} \frac{\left|\left(r^{\kappa} v\right)^{\prime}\right|^{2}}{r^{2 \kappa}\left(1+\lambda-\phi_{\mathrm{el}}\right)}+\left(\phi_{\mathrm{el}}+1-\lambda\right)|v|^{2} d r
$$

Both terms inside the integral decrease in $\lambda$ so, if $v$ is regular enough, there is a unique $\lambda=\lambda(v) \in \mathbb{R}$ satisfying $A(\lambda) v=0$. Upper estimates for the eigenvalues of $H_{\kappa}$ are then found from those of the matrix corresponding to the $\lambda$-dependent form $A(\lambda) v$ restricted to $v \in \mathcal{M}_{n}$.

REMARK 1. In the above we characterise the eigenvalues of (2.3) by detecting whether 0 lies in the discrete spectrum of auxiliary semi-bounded operator, $L_{E}$, depending on a scalar parameter. The method discuss in the forthcoming section resembles this approach. Rather than $L_{E}$, we introduce the operator polynomial $\left(z-H_{\kappa}\right)^{2}$. For each $z \in \mathbb{C}$, we see that $\left(z-H_{\kappa}\right)^{2}$ is a sectorial operator. We will see below that, when $z$ is close to an eigenvalue of $H_{\kappa}$, a neighbourhood of 0 is always protected against spectral pollution (see also the discussion preceding [5, Lemma 5]). Therefore, estimates for the eigenvalues of $H_{\kappa}$ will be found from those of the finite-dimensional operator polynomial $\left(z-H_{\kappa}\right)^{2}$ restricted to $\mathcal{L}_{M N}$. Condition (2.9) will be substituted by (3.14).

\section{The quadratic projection method}

\subsection{The second-order relative spectrum}

We now describe the basics of the quadratic projection method. In order to simplify the notation, below and elsewhere $G$ denotes a generic self-adjoint operator with domain $\operatorname{Dom}(G)$ acting on a Hilbert space. One should regard $G$ as being any of the $H_{\kappa}$ introduced in the previous section. The inner product in this Hilbert space is denoted by $\langle\cdot, \cdot\rangle$ and the norm by $\|\cdot\|$.

Let $\mathcal{L} \subset \operatorname{Dom}(G)$ be a subspace of finite dimension. Assume that

$$
\mathcal{L}=\operatorname{Span}\left\{b_{1}, \ldots, b_{n}\right\}
$$

where the vectors $b_{j}$ are linearly independent. Let

$$
K:=\left(\left\langle G b_{j}, G b_{k}\right\rangle\right)_{j, k=1}^{n}, \quad L:=\left(\left\langle G b_{j}, b_{k}\right\rangle\right)_{j, k=1}^{n} \quad \text { and } \quad B:=\left(\left\langle b_{j}, b_{k}\right\rangle\right)_{j, k=1}^{n} .
$$

For $z \in \mathbb{C}$, let $Q(z):=B z^{2}-2 z L+K \in \mathbb{C}^{n \times n}$. The aim of the quadratic projection method is to compute the so-called second-order spectrum of $G$ relative to $\mathcal{L}$ :

$$
\operatorname{Spec}_{2}(G, \mathcal{L}):=\operatorname{Spec}(Q)=\left\{\lambda \in \mathbb{C}: Q(\lambda) \underline{v}=0, \text { some } 0 \neq \underline{v} \in \mathbb{C}^{n}\right\} .
$$

Since $B$ is a non-singular matrix, $\operatorname{Spec}_{2}(G, \mathcal{L})$ consists of at most $2 n$ points. These points do not lie on the real line, except if $\mathcal{L}$ contains eigenvectors of $G$. However, since $Q(z)^{*}=Q(\bar{z})$, we have that

$$
\overline{\operatorname{Spec}_{2}(G, \mathcal{L})}=\operatorname{Spec}_{2}(G, \mathcal{L}) .
$$

The approximation of the discrete spectrum of $G$ using the second-order spectrum has been discussed in $[\mathbf{6}, \mathbf{7}, \mathbf{1 4}]$ and the references therein.

The following result establishes a crucial connection between $\operatorname{Spec}(G)$ and $\operatorname{Spec}_{2}(G, \mathcal{L})$. Without further mention, we often identify the elements $v \in \mathcal{L}$ with the corresponding $\underline{v} \in \mathbb{C}^{n}$ in the obvious manner:

$$
v=\sum_{k=1}^{n}\left\langle v, b_{j}^{*}\right\rangle b_{j} \quad \text { and } \quad \underline{v}=\left(\left\langle v, b_{j}^{*}\right\rangle\right)_{j=1}^{n} \text {, }
$$


where $\left\{b_{j}^{*}\right\}$ is the basis conjugate to $\left\{b_{j}\right\}$. Note that if the $b_{j}$ are mutually orthogonal and $\left\|b_{j}\right\|=1$, then $b_{j}^{*}=b_{j}$. Below and elsewhere $\Pi_{\mathcal{S}}$ denotes the orthogonal projection onto a subspace $\mathcal{S} \subset \operatorname{Dom}(G)$.

Theorem 1. Let $\mathcal{L} \subset \operatorname{Dom}(G)$ be finite-dimensional. If $\lambda \in \operatorname{Spec}_{2}(G, \mathcal{L})$, then

$$
[\operatorname{Re}(\lambda)-|\operatorname{Im}(\lambda)|, \operatorname{Re}(\lambda)+|\operatorname{Im}(\lambda)|] \cap \operatorname{Spec}(G) \neq \varnothing .
$$

Moreover, suppose that $E$ is an isolated eigenvalue of $G$ with associated eigenspace $\mathcal{E} \subset$ $\operatorname{Dom}(G)$. Let

$$
d_{E}:=\operatorname{dist}(E, \operatorname{Spec} G \backslash\{E\})=\min \{|E-x|: x \in \operatorname{Spec} G, x \neq E\} .
$$

If

$$
[\operatorname{Re}(\lambda)-|\operatorname{Im}(\lambda)|, \operatorname{Re}(\lambda)+|\operatorname{Im}(\lambda)|] \cap \operatorname{Spec}(G)=\{E\}
$$

and $Q(\lambda) \underline{v}=0$ for $0 \neq \underline{v} \in \mathbb{C}^{n}$, then the corresponding $v \in \mathcal{L}$ satisfies

$$
\frac{\left\|v-\Pi_{\mathcal{E}} v\right\|}{\|v\|} \leqslant \frac{\sqrt{2}|\operatorname{Im} \lambda|}{d_{E}} .
$$

Proof. Let $\lambda \in \operatorname{Spec}_{2}(G, \mathcal{L})$. If $\lambda \in \mathbb{R}$, both conclusions of the theorem are obvious, so throughout the proof we assume that $\operatorname{Im}(\lambda) \neq 0$. Since

$$
Q(z)=\left(\left\langle(z-G) b_{j},(\bar{z}-G) b_{k}\right\rangle\right)_{j, k=1}^{n},
$$

we see that $Q(\lambda) \underline{v}=0$ for non-trivial $\underline{v} \in \mathbb{C}^{n}$ if and only if

$$
\langle(\lambda-G) v,(\bar{\lambda}-G) w\rangle=0 \quad \forall w \in \mathcal{L} .
$$

For $u, w \in \mathcal{L}$ and $z=\mu+i \nu$ where $\mu, \nu \in \mathbb{R}$, we have

$$
\langle(z-G) u,(\bar{z}-G) w\rangle=\langle(\mu-G) u,(\mu-G) w\rangle-\nu^{2}\langle u, w\rangle-2 i \nu\langle(\mu-G) u, w\rangle .
$$

In particular, if we take $w=v$ in (3.5), we achieve

$$
\|(\operatorname{Re} \lambda-G) v\|^{2}-|\operatorname{Im} \lambda|^{2}\|v\|^{2}-2 i|\operatorname{Im} \lambda|\langle(\operatorname{Re} \lambda-G) v, v\rangle=0 .
$$

Thus

$$
\frac{\|(\operatorname{Re} \lambda-G) v\|}{\|v\|}=|\operatorname{Im} \lambda|
$$

and

$$
|\operatorname{Im} \lambda|\langle(\operatorname{Re} \lambda-G) v, v\rangle=0 .
$$

But recall that $G=G^{*}$, so

$$
\operatorname{dist}(x, \operatorname{Spec} G)=\min _{u \in \operatorname{Dom}(G)} \frac{\|(x-G) u\|}{\|u\|}
$$

for $x \in \mathbb{R}$. Therefore

$$
\operatorname{dist}(\operatorname{Re} \lambda, \operatorname{Spec} G) \leqslant|\operatorname{Im} \lambda|
$$

confirming (3.2).

For the second part, assume that $\lambda, E$ and $\mathcal{E}$ are as in the hypothesis, and let $v$ satisfy (3.5) and hence (3.6). Since

$$
\operatorname{dist}(x, \operatorname{Spec} G \backslash\{E\})=\min _{u \in \operatorname{Dom}(H), u \perp \mathcal{E}} \frac{\|(x-G) u\|}{\|u\|},
$$


we have

$$
\left\|(E-G)\left(I-\Pi_{\mathcal{E}}\right) v\right\| \geqslant d_{E}\left\|\left(I-\Pi_{\mathcal{E}}\right) v\right\|
$$

so that

$$
\left\|\left(I-\Pi_{\mathcal{E}}\right) v\right\| \leqslant \frac{\|(E-G) v\|}{d_{E}}
$$

Now,

$$
\|(E-G) v\|^{2}=|E-\operatorname{Re} \lambda|^{2}\|v\|^{2}+\|(\operatorname{Re} \lambda-G) v\|^{2}+2(E-\operatorname{Re} \lambda)\langle(\operatorname{Re} \lambda-G) v, v\rangle .
$$

Thus, by (3.6) and (3.7),

$$
\|(E-G) v\|^{2}=|E-\operatorname{Re} \lambda|^{2}\|v\|^{2}+|\operatorname{Im} \lambda|^{2}\|v\|^{2} \leqslant 2|\operatorname{Im} \lambda|^{2}\|v\|^{2} .
$$

This gives (3.4).

The above theorem suggests a method for estimating $\operatorname{Spec}(G)$ from the points in $\operatorname{Spec}_{2}(G, \mathcal{L})$. We call this method the quadratic projection method. Choose a suitable $\mathcal{L} \subset \operatorname{Dom}(G)$, find $Q(z)$ and compute $\operatorname{Spec}_{2}(G, \mathcal{L})$. Those $\lambda \in \operatorname{Spec}_{2}(G, \mathcal{L})$ which are close to $\mathbb{R}$ will necessarily be close to $\operatorname{Spec}(G)$, with a two-sided error given by $|\operatorname{Im}(\lambda)|$. Moreover, if $\lambda$ is close enough to an isolated eigenvalue $E$ of $G$, then

$$
|\operatorname{Re} \lambda-E| \leqslant|\operatorname{Im} \lambda|
$$

and a vector $0 \neq v \in \mathcal{L}$ such that $Q(\lambda) \underline{v}=0$ approaches the eigenspace associated to this eigenvalue with an error also determined by $|\operatorname{Im}(\lambda)|$. Note that we need not be concerned with the position of $E$ relative to the essential spectrum or any semi-definiteness condition imposed on $G$. The procedure is always free from spectral pollution.

REMARK 2. A stronger statement implying the first part of Theorem 1 can be found in $[\mathbf{1 4}$, Lemma 5.2]. In fact, for isolated points of the spectrum, the residual on the right of (3.8) can actually be improved to $2|\operatorname{Im}(\lambda)|^{2} / d_{E}$ for $|\operatorname{Im}(\lambda)|$ sufficiently small; see [7, Corollary 2.6]. However, note that this later estimate is less robust in the sense that $d_{E}$ is not known a priori.

\subsection{The Hermite basis}

In the forthcoming sections we apply the quadratic projection method to $G=H_{\kappa}$. Motivated by the results of [6, Section 3.4] on Schrödinger operators with a band gap essential spectrum, we construct finite-dimensional subspaces $\mathcal{L} \subset \operatorname{Dom}\left(H_{\kappa}\right)$ generated by Hermite functions.

Let the odd-order Hermite functions be defined by

$$
\Phi_{k}(r):=c_{2 k+1}^{-1} h_{2 k+1}(r) e^{-r^{2} / 2}, \quad r \geqslant 0, k \geqslant 0,
$$

where $h_{n}(r)$ are the Hermite polynomials and $c_{n}=\sqrt{2^{n-1} n ! \sqrt{\pi}}$ are normalisation constants. Let

$$
\mathcal{L} \equiv \mathcal{L}_{N M}:=\operatorname{Span}\left\{\left(\begin{array}{c}
\Phi_{1}(r) \\
0
\end{array}\right), \ldots,\left(\begin{array}{c}
\Phi_{N}(r) \\
0
\end{array}\right),\left(\begin{array}{c}
0 \\
\Phi_{1}(r)
\end{array}\right), \ldots,\left(\begin{array}{c}
0 \\
\Phi_{M}(r)
\end{array}\right)\right\}
$$

Below we might consider an imbalance between the number of basis elements in the first and second components, $N \neq M$. Without further mention, we often write $\mathcal{L}_{N} \equiv \mathcal{L}_{N N}$ and $\mathcal{L}_{n} \equiv \mathcal{L}_{N(n), M(n)}$ when $M$ and $N$ depend upon $n$.

We now recall some properties of $\Phi_{k}(r)$. The Hermite polynomials are defined by the identity

$$
h_{n}(z):=(-1)^{n} e^{z^{2}} \frac{d^{n}}{d z^{n}} e^{-z^{2}}, \quad n \in \mathbb{N} .
$$


They satisfy the recursive formulae

$$
\begin{gathered}
h_{n}^{\prime}(z)=2 n h_{n-1}(z), \\
h_{n+1}(z)=2 z h_{n}(z)-2 n h_{n-1}(z)
\end{gathered}
$$

and form an orthogonal set on the interval $(-\infty, \infty)$ with weight factor $e^{-z^{2}}$,

$$
\int_{-\infty}^{\infty} h_{m}(z) h_{n}(z) e^{-z^{2}} d z=2^{n} n ! \sqrt{\pi} \delta_{n m} .
$$

The generating function of this family of polynomials is

$$
\sum_{n=0}^{\infty} h_{n}(z) \frac{t^{n}}{n !}=e^{2 z t-t^{2}}
$$

Thus

$$
\sum_{n=0}^{\infty} h_{n}(0) \frac{t^{n}}{n !}=\sum_{n=0}^{\infty} \frac{(-1)^{n} t^{2 n}}{n !}
$$

so that

$$
h_{n}(0)= \begin{cases}0 & \text { for } n \text { odd } \\ \frac{(-1)^{n / 2} n !}{(n / 2) !} & \text { for } n \text { even. }\end{cases}
$$

The odd-order Hermite functions are the normalised wave functions of a harmonic oscillator,

$$
-\Phi_{k}^{\prime \prime}(r)+r^{2} \Phi_{k}(r)=(4 k+3) \Phi_{k}(r),
$$

subject to a Dirichlet boundary condition at the origin. They form an orthonormal basis of $L^{2}(0, \infty)$ and so $B=I$ in $(3.1)$.

The entries of the matrices $K$ and $L$ in (3.1) can also be found explicitly from known properties of the Hermite polynomials. The crucial terms for assembling these matrices are given by Tables 1 and 2 .

\subsection{Convergence}

The procedure described above is useful, provided we can find points of $\operatorname{Spec}_{2}\left(H_{\kappa}, \mathcal{L}\right)$ near the real axis. Here we formulate sufficient conditions on the sequence of subspaces $\mathcal{L}_{n}$, in order to guarantee the existence of a sequence $\lambda_{n} \in \operatorname{Spec}_{2}\left(H_{\kappa}, \mathcal{L}_{n}\right)$ accumulating at points of the discrete spectrum of $H_{\kappa}$. We then show that the sequence of subspaces (3.9) satisfies these conditions.

TABLE 1. Term $\left\langle H_{\kappa} \Psi_{k l}, \Psi_{j m}\right\rangle$.

\begin{tabular}{ccc}
\hline & $m=1$ & $m=2$ \\
\hline$l=1$ & $T_{1}+F_{1}$ & $T_{2}(k, j)+\kappa T_{3}$ \\
$l=2$ & $-T_{2}(k, j)+\kappa T_{3}$ & $-T_{1}+F_{1}$ \\
\hline
\end{tabular}

TABLE 2. Term $\left\langle H_{\kappa} \Psi_{k l}, H_{\kappa} \Psi_{j m}\right\rangle$.

\begin{tabular}{ccc}
\hline & $m=1$ & $m=2$ \\
\hline$l=1$ & $T_{1}+T_{4}+\kappa T_{5}(k, j)+\kappa T_{5}(j, k)$ & $-T_{2}(k, j)-T_{2}(j, k)+2 \kappa F_{3}$ \\
& $+\kappa^{2} T_{6}+2 F_{1}+F_{2}$ & $+F_{4}(k, j)-F_{4}(j, k)$ \\
$l=2$ & $-T_{2}(k, j)-T_{2}(j, k)+2 \kappa F_{3}$ & $T_{1}+T_{4}-\kappa T_{5}(k, j)-\kappa T_{5}(j, k)$ \\
& $-F_{4}(k, j)+F_{4}(j, k)$ & $+\kappa^{2} T_{6}-2 F_{1}+F_{2}$ \\
\hline
\end{tabular}


We firstly recall the following result [6, Theorem 2.1]. Note that $\mathcal{L}_{N M}$ are subspaces of $\operatorname{Dom}\left(H_{\kappa}^{2}\right)$ for all $\kappa$.

TheOrem 2. Let $E$ be an isolated eigenvalue of finite multiplicity of $G$ with associated eigenspace denoted by $\mathcal{E}$. Suppose that $\mathcal{L}_{n} \subset \operatorname{Dom}\left(G^{2}\right)$ is a sequence of subspaces such that

$$
\left\|G^{p}\left(u-\Pi_{n} u\right)\right\| \leqslant \delta(n)\|u\| \quad \forall u \in \mathcal{E}, p=0,1,2,
$$

where $\delta(n) \rightarrow 0$ as $n \rightarrow \infty$ is independent of $u$ and $p$. Then there exists $b>0$ and $\lambda_{n} \in$ $\operatorname{Spec}_{2}\left(G, \mathcal{L}_{n}\right)$, such that

$$
\left|\lambda_{n}-E\right|<b \delta(n)^{1 / 2}
$$

We now verify (3.14) for $G=H_{\kappa}$ and $\mathcal{L}_{n}$ as in Section 3.2. To this end we consider an argument similar to the one discussed in [6, Section 3.4] for the case of the crystalline non-relativistic Schrödinger operator.

Let

$$
A=\left(\begin{array}{cc}
\tilde{A} & 0 \\
0 & \tilde{A}
\end{array}\right)
$$

where $\tilde{A}=-\partial_{r}^{2}+r^{2}$ acting on $L^{2}(0, \infty)$, subject to Dirichlet boundary conditions at the origin. We fix the domain of $A$ to be

$$
\operatorname{Dom}(A)=\bigcup_{N \in \mathbb{N}} \mathcal{L}_{N}=\operatorname{Dom}(\tilde{A}) \oplus \operatorname{Dom}(\tilde{A}),
$$

so that $A=A^{*}, A$ has a compact resolvent and $\mathcal{L}_{N} \ominus \mathcal{L}_{N-1}$ are the eigenspaces of $A$.

For $f$ and $g$ regular enough, we have

$$
H_{\kappa}\left(\begin{array}{l}
f \\
g
\end{array}\right)=\left(\begin{array}{l}
p_{1} f+p_{2} g \\
p_{3} f+p_{4} g
\end{array}\right) \quad \text { and } \quad H_{\kappa}^{2}\left(\begin{array}{l}
f \\
g
\end{array}\right)=\left(\begin{array}{c}
q_{1} f+q_{2} g \\
q_{3} f+q_{4} g
\end{array}\right)
$$

where $p_{j}$ are linear polynomials and $q_{j}$ are quadratic polynomials in the variables $\partial_{r}, \phi_{\mathrm{sc}, \mathrm{el}, \mathrm{am}}$ and $\kappa / r$. Condition (3.14) is achieved by showing that both $H_{\kappa}$ and $H_{\kappa}^{2}$ are relatively bounded in the sense of operators with respect to $A$.

The following results can be easily extended to more general potentials. Here we only consider those of interest in our present discussion.

Lemma 3. Suppose that

$$
\phi_{\mathrm{sc}, \mathrm{el}, \mathrm{am}}=\chi_{\mathrm{sc}, \mathrm{el}, \mathrm{am}}+\psi_{\mathrm{sc}, \mathrm{el}, \mathrm{am}} \quad \text { where }\left\{\begin{array}{l}
\left|\chi_{\mathrm{sc}, \mathrm{el}, \mathrm{am}}(r)\right| \leqslant c r^{-1}, \forall r>0, \\
\psi_{\mathrm{sc}, \mathrm{el}, \mathrm{am}} \in L^{\infty}(0, \infty),
\end{array}\right.
$$

for some constant $c>0$. Then $\operatorname{Dom}(A) \subseteq \operatorname{Dom}\left(H_{\kappa}^{2}\right)$ and there exist constants $a, b>0$ such that

$$
\left\|H_{\kappa}^{p} v\right\| \leqslant a\|A v\|+b\|v\| \quad \forall v \in \operatorname{Dom}(A), p=0,1,2 .
$$

Proof. It is enough to check that the $H_{\kappa}^{p}$ are relatively bounded with respect to $-\partial_{r}^{2} I_{\mathbb{C}^{2}}$. This, on the other hand, is a straightforward consequence of Hardy's inequality.

By combining this lemma with Theorem 2 and Theorem 1, we achieve the following.

Corollary 4. Let $E$ be an isolated eigenvalue of $H_{\kappa}$ of finite multiplicity with associated eigenspace $\mathcal{E}$. Let $\mathcal{L}_{N M}$ be defined by (3.9). Suppose $\phi_{\mathrm{sc}, \mathrm{el}, \mathrm{am}}$ satisfy (3.16) and assume additionally that $\mathcal{E} \subset \operatorname{Dom}\left(A^{q_{\mathrm{u}}}\right) \oplus \operatorname{Dom}\left(A^{q_{1}}\right)$ for some $q_{\mathrm{u}}, q_{\mathrm{l}}>1$. There exist constants $b_{\mathrm{u}}, b_{\mathrm{l}}>0$ 
large enough, independent of $N$ or $M$ and not necessarily equal, such that we can always find a sequence $\lambda_{N M} \in \operatorname{Spec}_{2}\left(H_{\kappa}, \mathcal{L}_{N M}\right)$ satisfying

$$
\begin{aligned}
& \left|\lambda_{N M}-E\right|<b_{\mathrm{u}} N^{-\left(q_{1}-1\right) / 2}+b_{\mathrm{l}} M^{-\left(q_{1}-1\right) / 2} \text { and } \\
& \left\|\left(I-\Pi_{\mathcal{E}}\right) v_{N M}\right\|<b_{\mathrm{u}} N^{-\left(q_{\mathrm{u}}-1\right) / 2}+b_{\mathrm{l}} M^{-\left(q_{1}-1\right) / 2},
\end{aligned}
$$

where $v_{N M} \in \mathcal{L}_{M N}$ solves $Q\left(\lambda_{N M}\right) \underline{v_{N M}}=0$ with $\left\|v_{N M}\right\|=1$.

Proof. Let $v=\left(\begin{array}{l}v_{1} \\ v_{2}\end{array}\right) \in \mathcal{E}$ normalised by $\|v\|=1$. By virtue of Lemma 3 and Theorem 2 , the desired conclusion follows if we are able to find $b=b_{j}>0$ such that

$$
\sum_{k=n}^{\infty}\left|\left\langle\tilde{A}^{r} v_{j}, \Phi_{k}\right\rangle\right|^{2}<b n^{-2(q-r)}
$$

for $r=0,1$ and $j=1,2$. In order to show (3.18), note that the hypothesis $v_{j} \in \operatorname{Dom}\left(A^{q}\right)$ ensures

$$
\begin{aligned}
(2 n)^{2(q-r)} \sum_{k=n}^{\infty}\left|\left\langle\tilde{A}^{r} v_{j}, \Phi_{k}\right\rangle\right|^{2} & \leqslant \sum_{k=n}^{\infty}(4 k+3)^{2 q}\left|\left\langle v_{j}, \Phi_{k}\right\rangle\right|^{2} \\
& =\sum_{k=n}^{\infty}\left|\left\langle\tilde{A}^{q} v_{j}, \Phi_{k}\right\rangle\right|^{2} \rightarrow 0
\end{aligned}
$$

as $n \rightarrow \infty$.

Remark 3. Suppose that the number of degrees of freedom $M+N=2 n$ is fixed. If $q_{\mathrm{u}}=q_{\mathrm{l}}$ and $b_{\mathrm{u}}=b_{\mathrm{l}}=b$ is chosen large enough, the bound on the right-hand side of (3.17) is optimal at $(N, M)=(n, n)$. This suggests that an optimal rate of approximation might be achieved by choosing an equal number of upper/lower spinor components in (3.9). Contrary to this presumption, and depending on the potential $V$, the numerical evidence we present in Sections 4.4 and 4.5 shows that the residual on the right-hand side of (3.8) can in some cases decrease significantly (over $18 \%$ in some cases) by suitably choosing $N \neq M$.

We now explore precise conditions on the potential, in order to guarantee the hypothesis of Corollary 4.

Lemma 5. Let $\phi_{\mathrm{sc}, \mathrm{el}, \mathrm{am}} \in C^{\infty}(0, \infty)$ be such that $\phi_{\mathrm{sc}, \mathrm{el}, \mathrm{am}}(r) \rightarrow 0$ as $r \rightarrow \infty$. Assume additionally that $r \mapsto r^{\alpha} \phi_{\mathrm{sc}, \mathrm{el}, \mathrm{am}}(r)$ are locally bounded for some $\alpha \in(0,1)$. Let $H_{\kappa} u=E u$. For sufficiently small $a>0$,

$$
\left\|e^{a r} u\right\|_{H^{p}(0, \infty)}<\infty \quad \forall p \in \mathbb{N} .
$$

Proof. See [1, Corollary 3.1]. Let

$$
D_{\kappa}=\left(\begin{array}{cc}
1 & -\frac{d}{d r}+\frac{\kappa_{j}}{r} \\
\frac{d}{d r}+\frac{\kappa_{j}}{r} & -1
\end{array}\right) \quad \text { and } \quad V=\left(\begin{array}{cc}
\phi_{\mathrm{sc}}+\phi_{\mathrm{el}} & \phi_{\mathrm{am}} \\
\phi_{\mathrm{am}} & -\phi_{\mathrm{sc}}+\phi_{\mathrm{el}}
\end{array}\right) \text {. }
$$

For any $0<\varepsilon<\min |E \pm 1|$, we can always separate $V=V_{c}+V_{\varepsilon}$ where:

(a) $V_{c}$ is smooth, it has compact support and a singularity of order $O\left(r^{-\alpha}\right)$ at the origin,

(b) $V_{\varepsilon}$ is smooth with bounded derivatives and $\left\|V_{\varepsilon}\right\|_{\infty}<\varepsilon$.

Then

$$
u=-\left(D_{\kappa}+V_{\varepsilon}-E\right)^{-1} V_{c} u .
$$

Multiplying this identity by $D_{\kappa}^{p}$ and $e^{a r}$ yields

$$
D_{\kappa}^{p} e^{a r} u=-D_{\kappa}^{p}\left(D_{\kappa}+V_{\varepsilon}+i a-E\right)^{-1} e^{a r} V_{c} u .
$$


Let $\sigma \in \mathbb{R}$. On the one hand, $\left(D_{\kappa}+V_{\varepsilon}+i a-E\right)^{-1}$ is a bounded operator from $H^{\sigma}(0, \infty)$ to $H^{\sigma+1}(0, \infty)$. On the other hand, multiplication by $V_{c}$ is a bounded operator from $H^{\sigma}(0, \infty)$ into $H^{\sigma-\alpha}(0, \infty)$. Indeed, note that [20, Theorem 2.1(i) for $\left.p=q=2\right]$ yields the latter result for $\sigma \in$ $\mathbb{N}$ after commutation and iteration, and then duality and interpolation ensure it for all $\sigma \in \mathbb{R}$. Therefore, as $\left(D_{\kappa}+V_{\varepsilon}+\mathrm{i} a-E\right)^{-1} V_{c}$ is bounded from $H^{\sigma}(0, \infty)$ to $H^{\sigma+(1-\alpha)}(0, \infty),(3.19)$ and a standard bootstrap argument ensure the desired conclusion.

We remark that, by using [11, Lemma 5.1] (which can be modified in order to get rid of the term $\frac{1}{2}$ in its assumption $(i)$ ), necessarily $a<\sqrt{(1-\varepsilon)^{2}-E^{2}}$ for the above lemma to hold true.

Corollary 6. Assume that $\phi_{\mathrm{sc}, \mathrm{el}, \mathrm{am}}$ are as in Lemma 5. Let $E$ be an isolated eigenvalue of $H_{\kappa}$ of finite multiplicity with associated eigenspace $\mathcal{E}$. Let $\mathcal{L}_{N M}$ be defined by (3.9). There exist $b_{\mathrm{u}}, b_{\mathrm{l}}>0$ independent of $N$ or $M$, and a sequence $\lambda_{N M} \in \operatorname{Spec}_{2}\left(H_{\kappa}, \mathcal{L}_{N M}\right)$, such that (3.17) holds true for $1<q_{\mathrm{u}}=q_{1}<5 / 4$.

Proof. Let $v \in \mathcal{E}$ be as in the proof of Corollary 4. Let $q_{\mathrm{u}}=q_{\mathrm{l}}=q$. We show that

$$
\sum_{k=0}^{\infty}(4 k+3)^{2 q}\left|\left\langle v_{j}, \Phi_{k}\right\rangle\right|^{2}<\infty,
$$

for $j=1,2$. Let $F=\left(\partial_{r}-r\right)$. Integration by parts and (3.10) yield

$$
\begin{aligned}
\left\langle v_{j}, \Phi_{k}\right\rangle= & \frac{1}{c_{2 k+1}} \int_{0}^{\infty} v_{j}(r) h_{2 k+1}(r) e^{-r^{2} / 2} d r \\
= & \frac{1}{2(2 k+2) c_{2 k+1}} \int_{0}^{\infty} v_{j}(r) h_{2 k+2}^{\prime}(r) e^{-r^{2} / 2} d r \\
= & \frac{-1}{2(2 k+2) c_{2 k+1}} \int_{0}^{\infty} F v_{j}(r) h_{2 k+2}(r) e^{-r^{2} / 2} d r \\
= & \frac{1}{2^{2}(2 k+2)(2 k+3) c_{2 k+1}} \int_{0}^{\infty} F v_{j}(r) h_{2 k+3}^{\prime}(r) e^{-r^{2} / 2} d r \\
= & \frac{1}{2^{2}(2 k+2)(2 k+3) c_{2 k+1}} \int_{0}^{\infty} F^{2} v_{j}(r) h_{2 k+3}(r) e^{-r^{2} / 2} d r \\
= & \frac{-1}{2^{3}(2 k+2)(2 k+3)(2 k+4) c_{2 k+1}} \\
& \times\left(F^{2} v_{j}(0) h_{2 k+4}(0)+\int_{0}^{\infty} F^{3} v_{j}(r) h_{2 k+4}(r) e^{-r^{2} / 2} d r\right) \\
= & \frac{-F^{2} v_{j}(0) h_{2 k+4}(0)}{2^{3}(2 k+2)(2 k+3)(2 k+4) c_{2 k+1}}+\frac{\int_{0}^{\infty} F^{4} v_{j}(r) h_{2 k+5}(r) e^{-r^{2} / 2} d r}{2^{4}(2 k+2) \ldots(2 k+5) c_{2 k+1}} \\
= & a_{1}+a_{2} .
\end{aligned}
$$

Identity (3.12) alongside the fact that $\left|F^{2} v_{j}(0)\right|<\infty$ (see Lemma 5) and the Stirling formula ensures that $a_{1} \sim k^{-7 / 4}$ as $k \rightarrow \infty$. On the other hand, Lemma 5 ensures that $F^{4} v_{j} \in L^{2}(0, \infty)$. Thus, since

$$
\frac{c_{2 k+5}}{2^{4}(2 k+2) \ldots(2 k+5) c_{2 k+1}} \sim k^{-2}
$$

$a_{2}=O\left(k^{-2}\right)$ as $k \rightarrow \infty$. This guarantees (3.20) for $1<q<5 / 4$. 
According to this corollary, for smooth potentials the order of approximation of the quadratic projection method to any eigenvalue $E$ of $H_{\kappa}$ should be at least a power $1 / 8$ of the dimension of $\mathcal{L}_{M N}$. The numerical experiment performed in Section 3.3 shows that this bound improves substantially for particular potentials. See Figure 8 (right) and Table 5 (right).

REMARK 4. The arguments involved in the proof of this theorem show that the behaviour of the wave functions at the singularity and its regularity are the main ingredients responsible for controlling the speed of approximation when using a Hermite basis (3.9).

\section{Some numerical experiments}

We now report on various numerical experiments performed for very simple radially symmetric potentials. It is not our intention to show accurate computations, but rather to illustrate how the method discussed in Section 3 can be implemented in order to rigorously enclose eigenvalues and compute eigenfunctions of the Dirac operator.

\subsection{Ground state of the purely coulombic potential}

We begin by considering the analytically solvable case of $V$ being a radially symmetric purely coulombic potential: $\phi_{\mathrm{sc}}=\phi_{\mathrm{am}}=0$ and $\phi_{\mathrm{el}}(r)=\gamma / r$. Here

$$
-\sqrt{3} / 2<\gamma<0
$$

This case is not covered by Corollary 4 .

The eigenvalues of $H_{\kappa}$ are given explicitly by

$$
E_{j}=\left(1+\frac{\gamma^{2}}{\left(j+\sqrt{\kappa^{2}-\gamma^{2}}\right)^{2}}\right)^{-1 / 2} .
$$

Note that $E_{j} \rightarrow 1$ as $j \rightarrow \infty$ for all values of $\kappa$. The ground state of the full coulombic Dirac operator $H$ is achieved when $\kappa=-1$ and $j=0$.

In Figure 1 we superimpose the computation of $\operatorname{Spec}_{2}\left(H_{-1}, \mathcal{L}_{n}\right)$ for two values of $n$ in a narrow box near the interval $[-3,3]$. For the set of parameters considered $(\kappa=-1$ and $\gamma=-1 / 2)$, (4.1) yields $E_{0} \approx 0.866025, \quad E_{1} \approx 0.965925, \quad E_{2} \approx 0.9851210, \quad E_{3} \approx 0.99174012$ and $E_{4} \approx$ 0.9947623 . A two-sided approximation of $E_{0}$ is achieved from the point $\lambda \in \operatorname{Spec}_{2}\left(H_{-1}, \mathcal{L}_{1000}\right)$ at $\lambda \approx 0.8661+0.0236 i$. According to $(3.2)$, there should be an eigenvalue of $H_{-1}$ in the interval

$$
[0.8661-0.0236,0.8661+0.0236] \text {. }
$$

This eigenvalue happens to be $E_{0}$. For $E_{1}, E_{2}$ and the pair $\left(E_{3}, E_{4}\right)$, we can also derive similar conclusions. Note that $\operatorname{Spec}_{\text {ess }}\left(H_{-1}\right)$ is also revealed by points of $\operatorname{Spec}_{2}\left(H_{-1}, \mathcal{L}_{n}\right)$ seemingly accumulating at $(-\infty,-1] \cup[1, \infty)$.

In Figure 2 we show an approximation of the corresponding ground wave function associated with $E_{0}$. We have also depicted the analytical eigenfunction:

$$
u_{0}(r)=\nu_{0}\left(\begin{array}{c}
\gamma \\
\left(1-\gamma^{2}\right)^{1 / 2}-1
\end{array}\right) r^{\sqrt{1-\gamma^{2}}} e^{-\left(\gamma E_{0} / \sqrt{1-\gamma^{2}}\right) r},
$$

where $\nu_{0}$ is chosen so that $\left\|u_{0}\right\|=1$. From this figure it is clear that, at least qualitatively, $u_{0}(r)$ seems to be captured quite well even for small values of $n$.

We show a quantitative analysis of the calculation of $u_{0}$ in Table 3 . In the middle column we compute the residual on the left-hand side of (3.4) and on the last column we compute the right-hand side of (3.4). It is quite remarkable that the actual residuals are over $74 \%$ smaller than the error predicted by Theorem 1. 

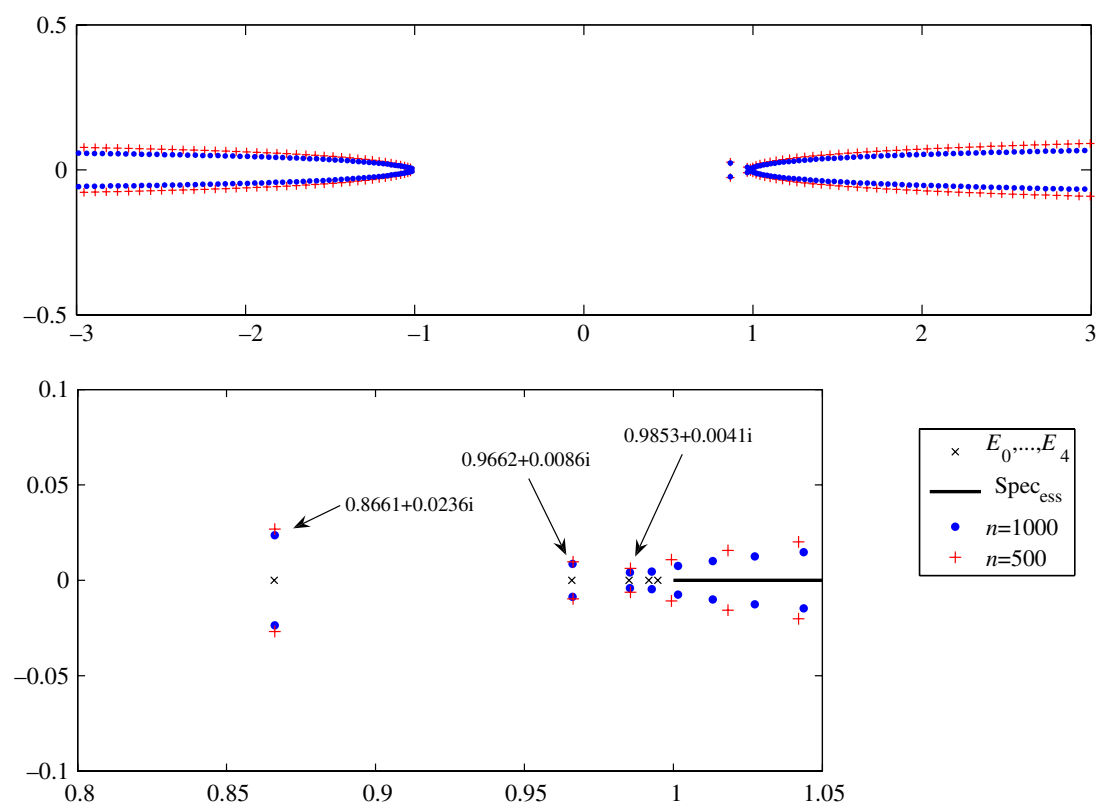

Figure 1. Portion of $\operatorname{Spec}_{2}\left(H_{-1}, \mathcal{L}_{n}\right)$ in thin boxes around $[-3,3]$ for the purely coulombic potential with $\gamma=-1 / 2$. The bottom graph shows details of the top picture near 1 . Here we superimpose two values of $n, 500$ and 1000 . According to Theorem 1 there are approximate energy states at $E \approx 0.8661 \pm 0.0236, E \approx 0.9662 \pm 0.0086$ and $E \approx 0.9853 \pm 0.0041$. These correspond to the actual eigenvalues $E_{0} \approx 0.866025, E_{1} \approx 0.965925$ and $E_{2} \approx 0.985121$.

\section{2. $\beta$-dependence of the sub-coulombic potential}

We now investigate the case of the potential being radially symmetric and sub-coulombic: $\phi_{\mathrm{sc}}=\phi_{\mathrm{am}}=0$ and $\phi_{\mathrm{el}}(r)=\gamma / r^{\beta}$ for $\beta \in(0,1)$. Here $-1<\gamma<0$. The purpose of this experiment is to show how Theorem 1 provides a priori information about $\operatorname{Spec}_{\text {disc }}\left(H_{\kappa}\right)$ even for small values of $n$. Note that (1.2) is guaranteed from [19, Theorems 4.7 and 4.17]. Furthermore, $H$ has infinitely many eigenvalues according to [19, Theorem 4.23].

In Figure 3, we show the computation of the ground state of $H_{-1}$ for $\beta=0.1: 0.1: 1$ and $\gamma=-1 / 2$. As $\beta \rightarrow 1^{-}$, we see that $E_{0} \rightarrow 0.8661$, the ground eigenvalue of the coulombic Dirac operator. As $\beta \rightarrow 0^{+}$the eigenvalue remains above $1 / 2$. Note that the family of operators $H_{\kappa}$ is not analytic at $\beta=0$ for this potential. For $\beta=0$ the spectrum becomes

$$
\operatorname{Spec}\left(H_{\kappa}\right)=(-\infty,-3 / 2] \cup[1 / 2, \infty) .
$$

The vertical bars show $|\operatorname{Im}(\lambda)|$, the maximum error in the computation of $E_{0} \approx \operatorname{Re}(\lambda)$ given by Theorem 1 . For this example we have chosen $n=15$. Table 4 contains the data depicted in Figure 2. Observe that the error increases as $\beta \rightarrow 0^{+}$and $\beta \rightarrow 1^{-}$. This seems to be a consequence of the fact that $E_{0}$ becomes closer to other spectral points at both limits, for instance $d_{E_{0}} \rightarrow 0$ as $\beta \rightarrow 0^{+}$.

TABLE 3. Here we compare both sides of (3.4) for the computation of the approximate eigenfunctions of Figure 2. We approximate $d_{E}=E_{1}-E_{0} \approx 0.0999004$.

\begin{tabular}{lcc}
\hline$n$ & $\left\|v-\Pi_{\mathcal{E}} v\right\| /\|v\|$ & $\sqrt{2}|\operatorname{Im}(\lambda)| / d_{E}$ \\
\hline 15 & 0.176115 & 0.962512 \\
25 & 0.084527 & 0.727195 \\
35 & 0.072552 & 0.646343 \\
\hline
\end{tabular}



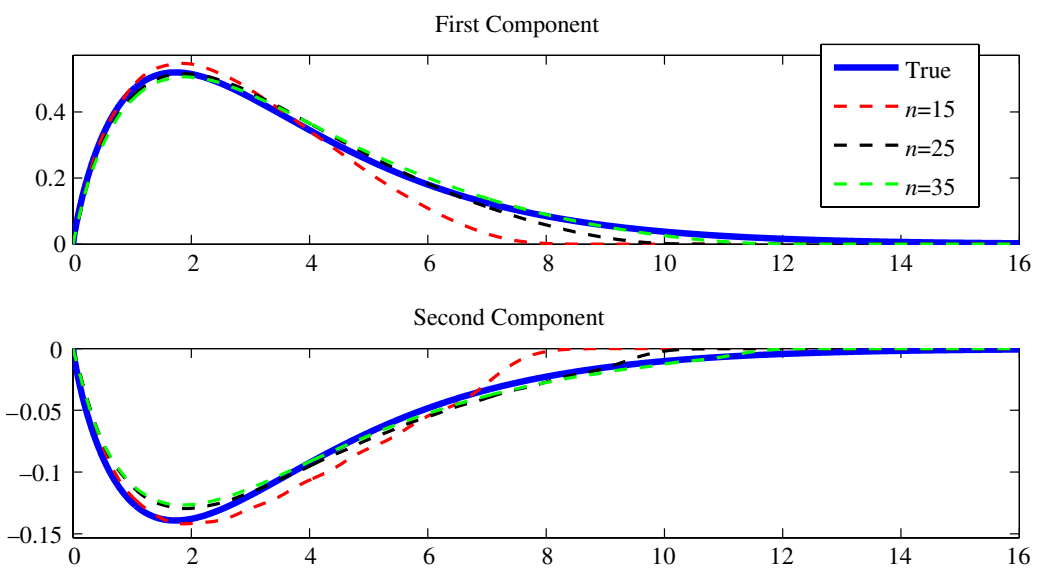

Figure 2. Approximate ground wave function for a purely coulombic potential with $\gamma=-1 / 2$. The true wave function (blue line) can be found explicitly, [19, Section 7.4.2]. The ground state in this case is $E_{0}=\sqrt{1-\gamma^{2}} \approx 0.866025$. We have deliberately chosen small dimensions $n$ for the test spaces, in order to illustrate the approximation of the method. The residual error is actually much smaller than that predicted by Theorem 1, see Table 3.

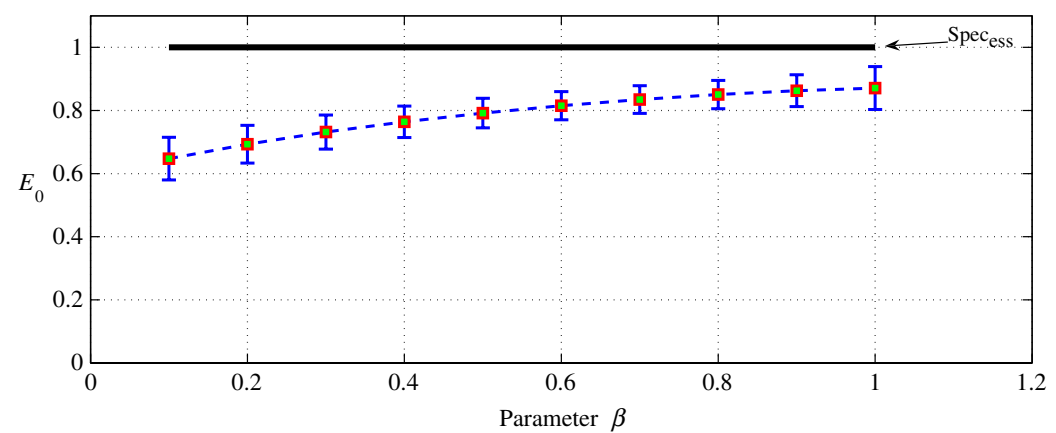

Figure 3. Computation of the ground energy value for $H_{-1}$ and $\phi_{\mathrm{el}}(r)=-1 /\left(2 r^{\beta}\right)$. We depict $E_{0}$ against $\beta$. The vertical bars correspond to the error predicted by Theorem 1.

\subsection{The inverse harmonic electric potential}

In this set of experiments we consider another canonical example in the theory of Dirac operators: $\phi_{\mathrm{sc}}=\phi_{\mathrm{am}}=0$ and $\phi_{\mathrm{el}}(r)=\gamma /\left(1+r^{2}\right)$ for $\gamma<0$. The discrete spectrum of $H$ is known to be finite for $-1 / 8<\gamma<0$ and infinite for $\gamma<-1 / 8[\mathbf{1 2}]$. As the parameter $\gamma$ decreases, we

TABle 4. Data depicted in Figure 3.

\begin{tabular}{ccc}
\hline$\beta$ & $E_{0}$ & $|\operatorname{Im}(\lambda)|$ \\
\hline 0.1 & 0.6474 & 0.0675 \\
0.2 & 0.6932 & 0.0599 \\
0.3 & 0.7316 & 0.0542 \\
0.4 & 0.7642 & 0.0499 \\
0.5 & 0.7918 & 0.0468 \\
0.6 & 0.8151 & 0.0448 \\
0.7 & 0.8346 & 0.0439 \\
0.8 & 0.8505 & 0.0449 \\
0.9 & 0.8627 & 0.0504 \\
1.0 & 0.8711 & 0.0680 \\
\hline
\end{tabular}



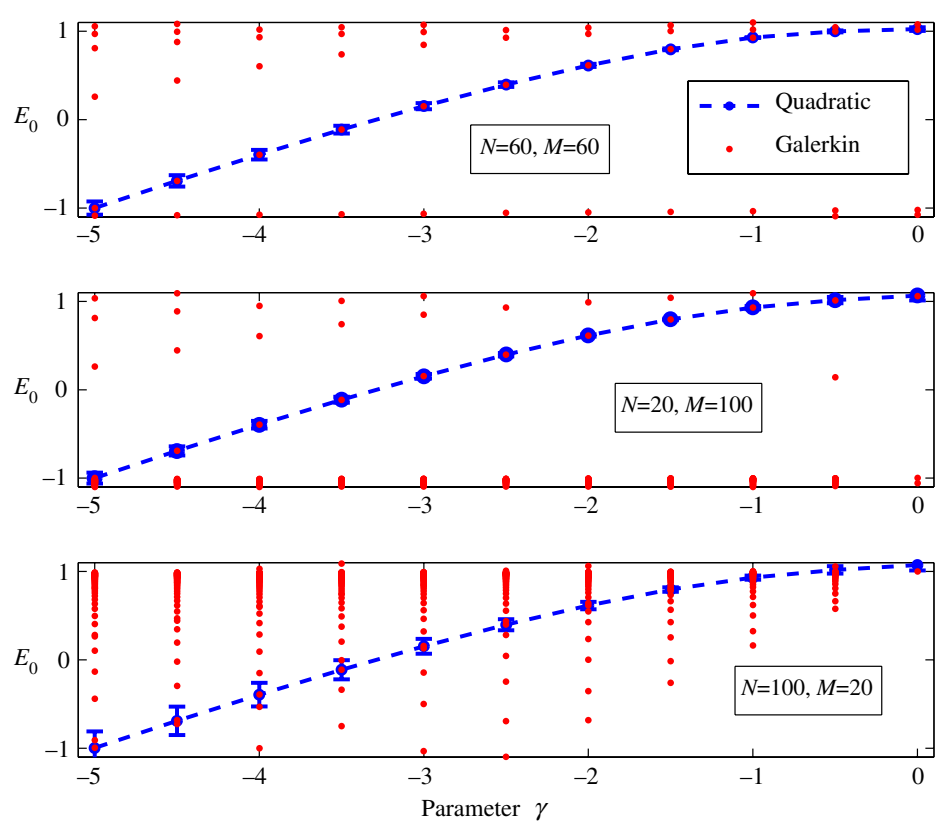

FiguRE 4. The graph captures the evolution of $E_{0}$ as it crosses the spectral gap of $H_{-1}$ where $\phi_{\mathrm{el}}(r)=\gamma /\left(1+r^{2}\right)$ for $\gamma=-5: 0.5: 0$. We consider three choices of pairs $(N, M)$ such that $\operatorname{dim}\left(\mathcal{L}_{N M}\right)=120$. The curve corresponds to $\operatorname{Re}\left(\lambda_{n}\right)$ for $\lambda=\lambda_{n}$ in (3.4) and (3.15). The vertical bars on the curve measure $\left|\operatorname{Im}\left(\lambda_{n}\right)\right|$. We superimpose the image with the eigenvalues of $L$ in (3.1) for $G=H_{-1}$, that are obtained by the Galerkin method.

expect that eigenvalues will appear at the threshold 1, move through the gap, and leave it at -1 . This dynamics is shown in Figure 4, for the ground eigenvalue of $H_{-1}$. It is a long-standing question whether the eigenvalues become resonances when they re-enter the spectrum.

In Figure 5 we depict the first three eigenfunctions of $H_{-1}$ for $\gamma=-4$. They correspond to the eigenvalues $E_{0} \approx-0.3955, E_{1} \approx 0.6049$ and $E_{2} \approx 0.9328$. See also Figure 7 . Note that for $\gamma=-4$ both components of the eigenfunctions appear to obey a Sturm-Liouville-type oscillation hierarchy.

\subsection{Upper/lower spinor component balance and approximation of eigenvalues}

We now investigate the effects of 'unbalancing' the basis by choosing $N \neq M$.

In Figure 6, we have performed the following experiment. Fix the number of degrees of freedom, $\operatorname{dim}\left(\mathcal{L}_{M N}\right)=200$. Then for $N=10: 5: 190$ and $M=200-N$, use the quadratic method

TABLE 5. In this table we fit by least squares the data of Figure 8 (left and right) and find $a$ and $b$ such that $\left|\lambda_{n}-E_{0}\right| \leqslant\left|\operatorname{Im}\left(\lambda_{n}\right)\right| \sim b n^{a}$ for $n=N+M$.

\begin{tabular}{ccclcc}
\hline$N$ & $a$ & $b$ & $N$ & $a$ & $b$ \\
\hline$n / 8$ & -0.6736 & 1.6766 & $n / 8$ & -1.3241 & 8.8276 \\
$n / 4$ & -0.5426 & 0.6555 & $n / 4$ & -0.9135 & 1.1303 \\
$3 n / 8$ & -0.4385 & 0.3530 & $3 n / 8$ & -0.7990 & 0.7223 \\
$n / 2$ & -0.3963 & 0.2703 & $n / 2$ & -0.7979 & 0.8155 \\
$5 n / 8$ & -0.5064 & 0.4478 & $5 n / 8$ & -0.8125 & 1.0825 \\
$3 n / 4$ & -0.6903 & 1.1115 & $3 n / 4$ & -0.8163 & 1.5171 \\
$7 n / 8$ & -0.9609 & 5.4520 & $7 n / 8$ & -0.8004 & 2.4558 \\
\hline
\end{tabular}



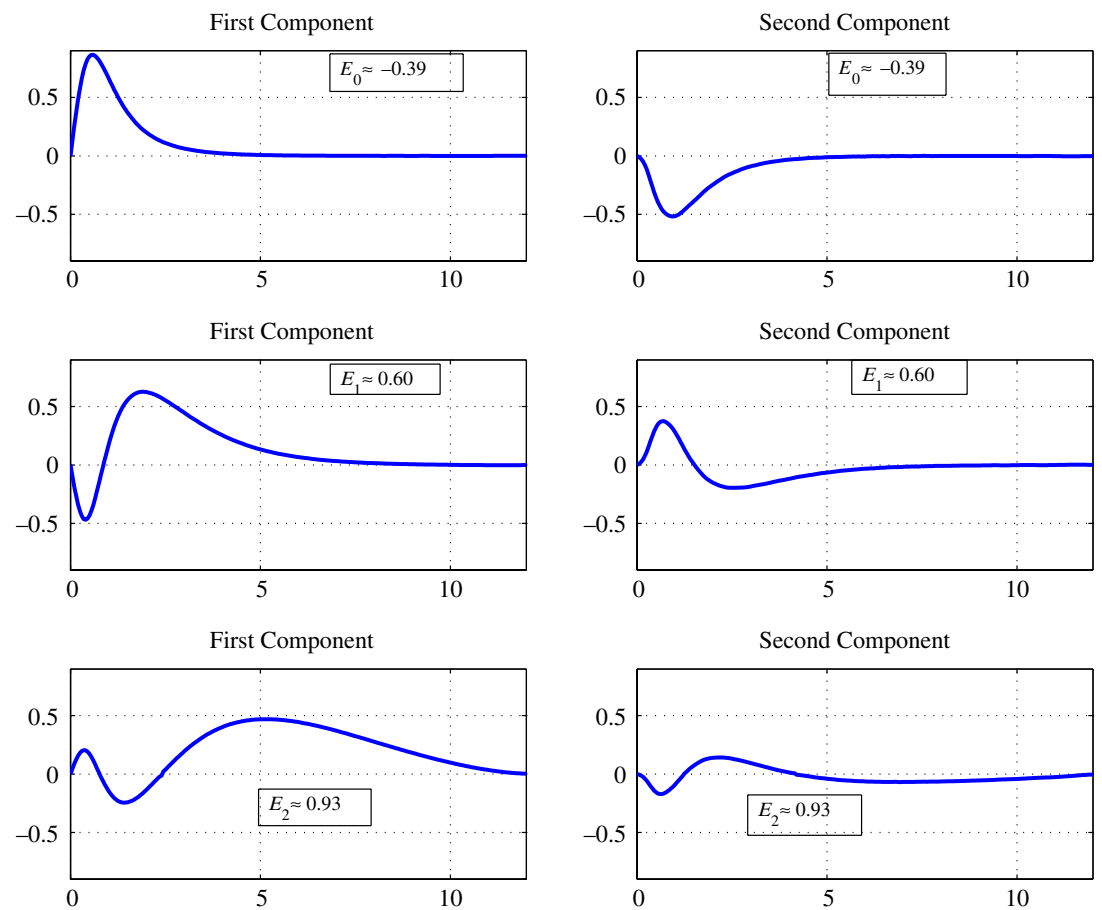

Figure 5. Here we use Theorem 1 to find the first three eigenfunctions of $H_{-1}$ with $\phi_{\mathrm{el}}(r)=-4 /\left(1+r^{2}\right)$. The numerical evidence suggests $E_{0} \approx-0.3955, E_{1} \approx 0.6049$ and $E_{2} \approx 0.9328$.
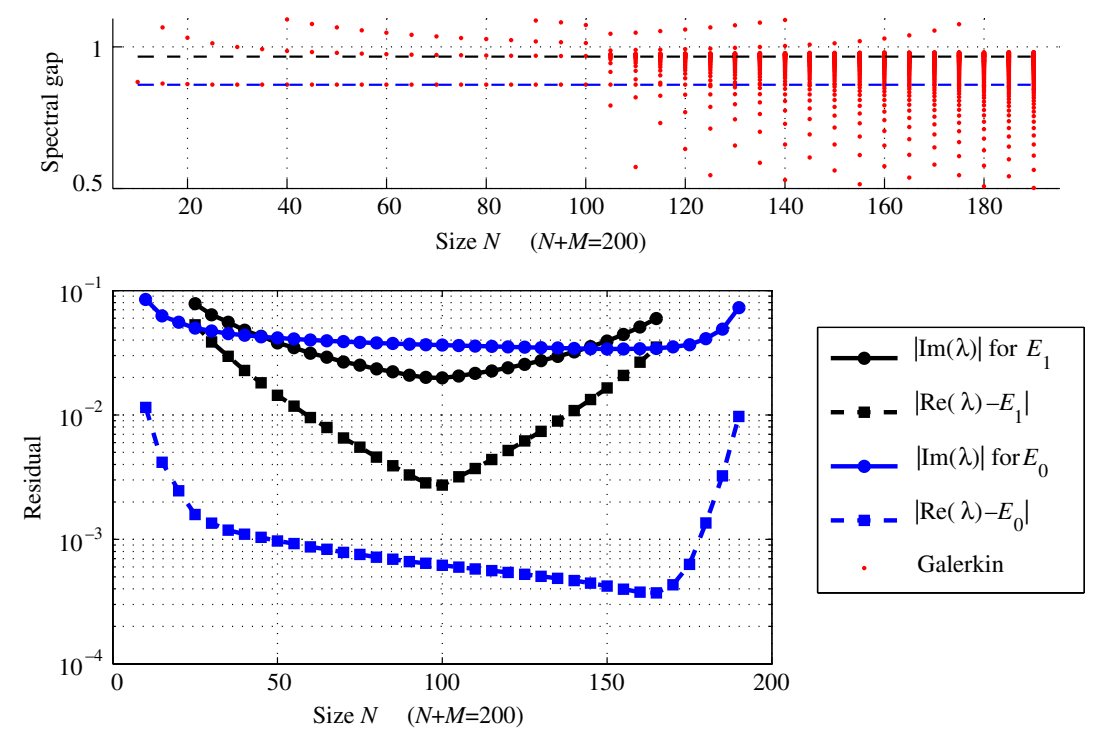

Figure 6. Here $E_{0}$ and $E_{1}$ are eigenvalues of $H_{-1}$ for $\phi_{\mathrm{el}}(r)=-1 / 2 r$. The top graph shows the eigenvalues of $L$ in (3.1) (that is, the Galerkin approximation) for $G=H_{-1}$ and $(M, N)=(N, 200-N)$ so that $\operatorname{dim}\left(\mathcal{L}_{N M}\right)=200$. The bottom graph depicts the residuals $|\operatorname{Im}(\lambda)|$ and $\left|\operatorname{Re}(\lambda)-E_{j}\right|$. For $E_{0}$, the minimum of the residual curve corresponding to $|\operatorname{Im}(\lambda)|$ is achieved

when $N \approx 155$ and it is roughly $7 \%$ smaller than when $N=100$. For the same eigenvalue, the residual curve corresponding to $\left|\operatorname{Re}(\lambda)-E_{0}\right|$ achieves its minimum when $N=165$ and it is roughly $66 \%$ smaller than when $N=100$. 

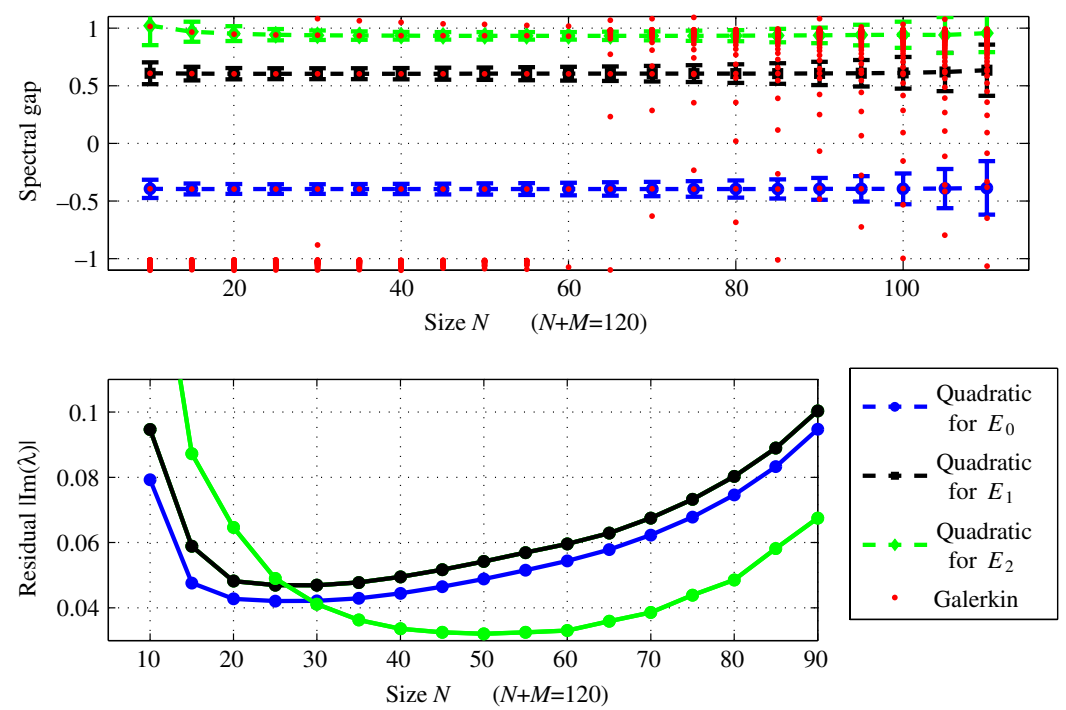

FiguRE 7. Here $E_{0}, E_{1}$ and $E_{2}$ are the first three eigenvalues of $H_{-1}$ for $\phi_{\mathrm{el}}(r)=-4 /\left(1+r^{2}\right)$. The top graph shows the approximation of $E_{0} \approx-0.3955, E_{1} \approx 0.6049$ and $E_{2} \approx 0.9328$ for $(M, N)=(N, 120-N)$ so that $\operatorname{dim}\left(\mathcal{L}_{N M}\right)=120$. The curves correspond to $\operatorname{Re}\left(\lambda_{n}\right)$ for $\lambda=\lambda_{n}$ in (3.4) and (3.15). The vertical bars measure $\left|\operatorname{Im}\left(\lambda_{n}\right)\right|$. The image is superimposed by the eigenvalues of $L$ in (3.1) for $G=H_{-1}$, that is, the Galerkin approximation. The bottom graph depicts the residuals $\left|\operatorname{Im}\left(\lambda_{n}\right)\right|$.
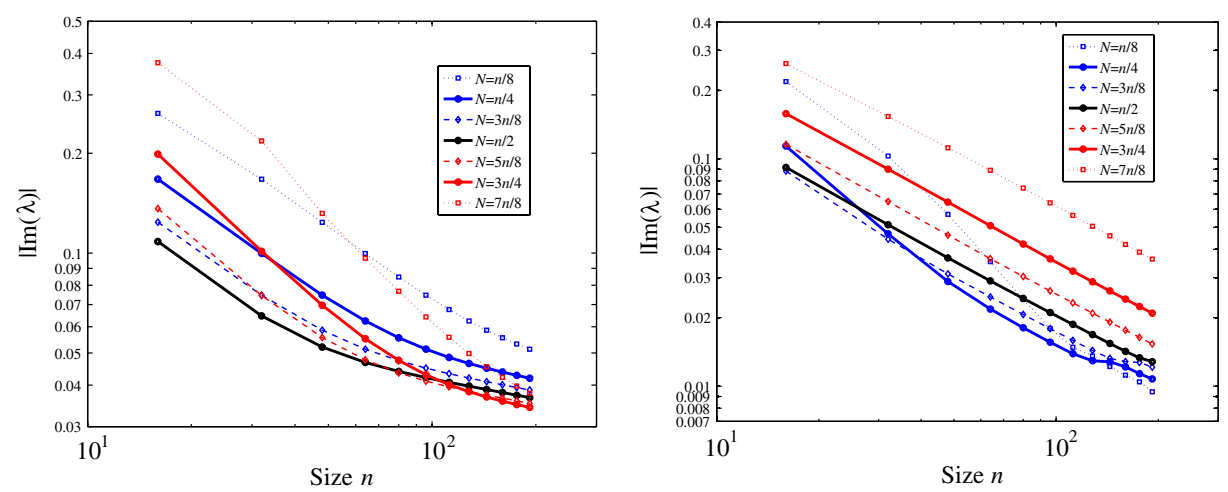

Figure 8. $\log -\log$ plots of $|\operatorname{Im}(\lambda)|$ for $\operatorname{Re}(\lambda)$ close to an eigenvalue, $E_{0}$, for different choices of pairs $(N, M)$ as $n=N+M$ increases. Left: $\kappa=-1, \phi_{\mathrm{el}}(r)=-1 / 2 r$ and $E_{0} \approx 0.86602$. Right: $\kappa=-1$, $\phi_{\mathrm{el}}(r)=-2 / 1+r^{2}$ and $E_{0} \approx 0.61399$. See Table 5.

as well as the Galerkin method to approximate eigenvalues of $H_{\kappa}$ in the spectral gap $(-1,1)$. We firstly consider $\phi_{\mathrm{sc}}=\phi_{\mathrm{am}}=0$ and $\phi_{\mathrm{el}}(r)=-1 /(2 r)$.

The Galerkin method might or might not produce spurious eigenvalues. The quadratic method will always provide two-sided non-polluted bounds for the true eigenvalues with a residual, obtained from (3.2), which might change with $N$. See also Figures 4 and 8 (left). The Galerkin method appears to pollute heavily near the upper end of the gap for $N>M$, as predicted by the considerations of Section 2. Moreover, for the ground state, the minimal $|\operatorname{Im}(\lambda)|$ is not achieved at $N=100$ which corresponds to $N=M$, but rather at some $N>100$. It is remarkable that the residuals are reduced significantly (up to $66 \%$ for the true residual) when $M(N) / N \approx 1 / 5$. 
If we perform the analogous experiment for the inverse harmonic potential, the conclusions are also rather surprising. See Figure 7. The Galerkin method appears to pollute heavily near the upper end of the gap for $N>M$ as predicted in Section 2. However, now the approximation is improved by over $16 \%$ for $E_{0}$ and over $18 \%$ for $E_{1}$, if $M(N) / N \approx 3$.

The above phenomena is related to the fact that the constants $b_{\mathrm{u}}$ and $b_{1}$ in Corollaries 4 and 6 do not need to be close to each other. This can be explained by considering the relation between the components of the exact eigenvectors.

In the case of a purely coulombic potential, the ground state is given by (4.2) where $\nu_{0}$ is a real constant. The lower spinor component just differs from the upper one by a scalar factor. When $\gamma \in(0,1)$, the lower component is smaller in modulus than the upper one. Choosing $N>M$ can reduce the upper bound of the residual associated to the first component, while the residual associated to the second component remains small due to the smallness of the lower component.

In the case of an inverse harmonic purely electric potential, this argument fails, as the two spinor components of the eigenfunction are not a scalar factor of each other; see Figure 5. If we denote an eigenfunction by $u=\left(u^{\mathrm{u}}, u^{\mathrm{l}}\right)$, the figure suggests that $\left|\partial_{r}^{2} u^{\mathrm{u}}(0)\right| \ll\left|\partial_{r}^{2} u^{\mathrm{l}}(0)\right|$. As $\left|\partial_{r}^{2} \Phi_{k}(0)\right|=0$, it is natural to expect that a decrease in the residual is only achieved by choosing a suitable $M>N$.

REMARK 5. Although we can not prove it rigorously, strong evidence suggests that (for any of the potentials considered above) no spurious eigenvalue is produced by the Galerkin method when $N=M$. Why bother then with more complicated procedures, such as the quadratic projection method, to avoid non-existent spectral pollution? A partial answer is, on the one hand, robustness: we do not know a priori whether the Galerkin method pollutes for a given basis. On the other hand, as the experiments of this section suggest, sometimes forcing pollution into a model might improve the convergence properties.

\subsection{Convergence properties of the odd Hermite basis}

A convergence analysis, as the number of degrees of freedom increases, can be found in Figure 8 and Table 5. Due to the discussion of Section 4.4, we consider different ratios $N / M$.

The right-hand graph shows that the conclusion of Corollary 6 is far from optimal for the inverse harmonic potential of Section 4.3. As expected from the discussion in Section 4.4, a faster convergence rate as well as smaller residuals are found if we suitably choose $N<M$.

The left-hand graph corresponds to the coulombic potential in Section 4.1. Note that the convergence rate seems to decrease as we increase the number of degrees of freedom. We suspect that this reduction in the speed of convergence can be prevented by putting $M=f(N)$ for a suitable non-linear increasing function $0<f(x)<x$. The optimal $f(x)$, however, might depend on the eigenvalue to be approximated.

REMARK 6. This provides a strong indication that the order of convergence of $\lambda_{n} \rightarrow E$ does not obey a residual estimate of the form $\left|\lambda_{n}-E\right| \leqslant O\left(n^{-a}\right)$ (for some $a>0$ ) in the case of coulombic potentials.

REMARK 7. According to Remark 2, the actual approximate eigenvalue $\operatorname{Re}(\lambda)$ is correct up to $O\left(n^{-2 a}\right)$, where $a$ is as in the second column of Table 5 . Furthermore, note that in the case of the coulombic potential we can compute directly the true residual $|\operatorname{Re}(\lambda)-E|$. From Figure 6 (bottom), it is clear that this true residual is substantially smaller than the one estimated by $|\operatorname{Im}(\lambda)|$. 


\section{Appendix. Entries of the matrix polynomial coefficients}

The recursive identities satisfied by the Hermite functions allow us to find recursive expressions for the matrix entries of $K$ and $L$ in (3.1) when $G=H_{\kappa}$. Rather than estimating the corresponding inner products by, for example, quadrature rules, we build the codes involved in the numerical experiments performed in Section 4 using these explicit expressions. As large factors are cancelled in these explicit expressions, this approach turns out to be far more accurate. Since some of the calculations are not entirely trivial, we include here the crucial details.

Let

$$
\begin{gathered}
T_{1}=\int_{0}^{\infty} \Phi_{k}(r) \Phi_{j}(r) d r, \quad T_{2}(k, j)=\int_{0}^{\infty} \Phi_{k}^{\prime}(r) \Phi_{j}(r) d r \\
T_{3}=\int_{0}^{\infty} \frac{1}{r} \Phi_{k}(r) \Phi_{j}(r) d r, \quad T_{4}=\int_{0}^{\infty} \Phi_{k}^{\prime}(r) \Phi_{j}^{\prime}(r) d r \\
T_{5}(k, j)=\int_{0}^{\infty} \frac{1}{r} \Phi_{k}^{\prime}(r) \Phi_{j}(r) d r, \quad T_{6}=\int_{0}^{\infty} \frac{1}{r^{2}} \Phi_{k}(r) \Phi_{j}(r) d r \\
F_{1}=\int_{0}^{\infty} \phi_{\mathrm{el}}(r) \Phi_{k}(r) \Phi_{j}(r) d r, \quad F_{2}=\int_{0}^{\infty} \phi_{\mathrm{el}}^{2}(r) \Phi_{k}(r) \Phi_{j}(r) d r \\
F_{3}=\int_{0}^{\infty} \frac{\phi_{\mathrm{el}}(r)}{r} \Phi_{k}(r) \Phi_{j}(r) d r, \quad F_{4}(k, j)=\int_{0}^{\infty} \phi_{\mathrm{el}}(r) \Phi_{k}^{\prime}(r) \Phi_{j}(r) d r .
\end{gathered}
$$

Here and below we stress the dependence on $j, k$ when the coefficient is not symmetric with respect to these indices. Denote

$$
\Psi_{k, 1}=\left(\begin{array}{c}
\Phi_{k} \\
0
\end{array}\right), \quad \Psi_{j, 2}=\left(\begin{array}{c}
0 \\
\Phi_{j}
\end{array}\right) .
$$

Then $\left\langle H_{\kappa} \Psi_{k l}, \Psi_{j m}\right\rangle$ are given according to Table 1 and $\left\langle H_{\kappa} \Psi_{k l}, H_{\kappa} \Psi_{j m}\right\rangle$ are given according to Table 2.

For $m, n \in \mathbb{N} \cup\{0\}$, let

$$
P(n)= \begin{cases}\prod_{l=1}^{n}\left(1+\frac{1}{2 l}\right), & n \neq 0 \\ 1, & n=0\end{cases}
$$

and

$$
I(m, n)=\frac{1}{c_{m} c_{n}} \int_{0}^{\infty} h_{m}(r) h_{n}(r) e^{-r^{2}} d r
$$

Lemma A.1. We have the following identities

$$
I(m, n)= \begin{cases}\delta_{m n}, & m \equiv n(\bmod 2), \\ \frac{(-1)^{k-j+1} \sqrt{2 P(k) P(j)}}{(2 k-2 j-1) \sqrt{\pi(2 k+1)}}, & m=2 k, n=2 j+1 .\end{cases}
$$

Proof. If $m \equiv n(\bmod 2)$, then $h_{m}(r) h_{n}(r)$ is an even function for $r \in \mathbb{R}$ and so

$$
\int_{0}^{\infty} h_{m}(r) h_{n}(r) e^{-r^{2}} d r=\frac{1}{2} \int_{-\infty}^{\infty} h_{m}(r) h_{n}(r) e^{-r^{2}} d r
$$


On the other hand, if $m \not \equiv n(\bmod 2)$, say $m=2 k$ and $n=2 j+1,(3.10)$ and integration by parts yield

$$
\begin{aligned}
\int_{0}^{\infty} h_{2 k}(r) h_{2 j+1}(r) e^{-r^{2}} d r & =\int_{0}^{\infty} h_{2 j+1}(r)\left(e^{-r^{2}}\right)^{(2 k)} d r \\
& =-\int_{0}^{\infty} h_{2 j+1}^{\prime}(r)\left(e^{-r^{2}}\right)^{(2 k-1)} d r \\
& =-2(2 j+1) \int_{0}^{\infty} h_{2 j}(r)\left(e^{-r^{2}}\right)^{(2 k-1)} d r \\
& =2^{2}(j+1)(2 k-1) \int_{0}^{\infty} h_{2 k-2}(r) h_{2 j-1}(r) e^{-r^{2}} d r
\end{aligned}
$$

The corresponding expression for $I(m, n)$ can be obtained in a straightforward manner from these two assertions.

LEMMA A.2. We have the following identities

$$
\begin{aligned}
& T_{1}(k, j)=\delta_{j k}, \quad T_{2}(k, j)=\frac{4(-1)^{k-j+1}(k-j)}{\sqrt{\pi}(2 k-2 j-1)(2 k-2 j+1)} \sqrt{P(j) P(k)}, \\
& T_{3}(k, j)=\frac{2(-1)^{k-j+1} \sqrt{P(j)}}{\sqrt{\pi} \sqrt{P(k)}} \sum_{m=0}^{k} \frac{P(m)}{(2 m+1)(2 m-2 j-1)}, \\
& T_{4}(k, j)=\frac{1}{2} \begin{cases}-\sqrt{2 k(2 k+1)}, & j=k-1 \\
4 k+3 & j=k \\
-\sqrt{(2 k+2)(2 k+3)}, & j=k+1 \\
0 & \text { otherwise }\end{cases} \\
& T_{5}(k, j)=\left\{\begin{array}{ll}
2(-1)^{j-k} \sqrt{\frac{P(k)}{P(j)}}, & k<j, \\
1, & k=j, \\
0, & k>j,
\end{array} \quad T_{6}(k, j)=(-1)^{j-k} 2 \begin{cases}\sqrt{\frac{P(j)}{P(k)}}, & j \leqslant k, \\
\sqrt{\frac{P(k)}{P(j)}}, & k<j .\end{cases} \right.
\end{aligned}
$$

Proof. Let $I(m, n)$ be given by (A.1). By virtue of identities (3.10) and (3.11),

$$
\begin{gathered}
\int_{0}^{\infty} h_{2 k+1}^{\prime} h_{2 j+1} e^{-r^{2}} d r=\sqrt{2(2 k+1)} c_{2 k+1} c_{2 j+1} I(2 k, 2 j+1), \\
\int_{0}^{\infty} \frac{1}{r} h_{2 k+1} h_{2 j+1} e^{-r^{2}} d r=\sum_{l=0}^{k}(-1)^{l} 2^{2 l+1} \frac{k !}{(k-l) !} c_{2(k-l)} c_{2 j+1} I(2(k-l), 2 j+1) .
\end{gathered}
$$

This yields $T_{2}$ and $T_{3}$.

Let

$$
J(k, j)=\int_{0}^{\infty} \frac{1}{r} h_{2 k} h_{2 j+1} e^{-r^{2}} d r=\frac{1}{2} \int_{-\infty}^{\infty} \frac{1}{r} h_{2 k} h_{2 j+1} e^{-r^{2}} d r .
$$

Then

$$
J(k, j)= \begin{cases}\frac{\sqrt{\pi} 2^{2 j}(2 k) !(-1)^{j-k} j !}{k !} & k \leqslant j \\ 0 & k>j\end{cases}
$$

and

$$
\int_{0}^{\infty} \frac{1}{r} h_{2 k+1}^{\prime} h_{2 j+1} e^{-r^{2}} d r=2(2 k+1) J(k, j)
$$


This renders $T_{5}$. Moreover, integration by parts ensures

$$
T_{6}=\int_{0}^{\infty} \frac{1}{r^{2}} \Phi_{k} \Phi_{j}=-\int_{0}^{\infty}\left(\frac{1}{r}\right)^{\prime} \Phi_{k} \Phi_{j}=\left(T_{5}(k, j)+T_{5}(j, k)\right) .
$$

The expression for $T_{4}$ follows from (3.13) and the identity

$$
\int_{0}^{\infty} \Phi_{k}^{\prime}(r) \Phi_{j}^{\prime}(r) d r=\frac{1}{2} \int_{-\infty}^{\infty}-\Phi_{k}^{\prime \prime}(r) \Phi_{j}(r) d r
$$

From $E_{1}$ and $E_{2}$ in the next lemma, one easily obtains explicit formulae for $F_{n}$ when $\phi_{\mathrm{el}}(r)=\gamma / r^{\beta}$.

Lemma A.3. For $\beta \in[0,1]$ and $\alpha \in[-1,2]$, let

$$
E_{1}(\beta, k, j)=\int_{0}^{\infty} \frac{1}{r^{\beta}} \Phi_{k}^{\prime}(r) \Phi_{j}(r) d r, \quad E_{2}(\alpha, k, j)=\int_{0}^{\infty} \frac{1}{r^{\alpha}} \Phi_{k}(r) \Phi_{j}(r) d r .
$$

Then

$$
E_{1}(\beta, k, j)=(2 k+1) E_{2}(\beta+1, k, j)+\sqrt{2 k(2 k+1)} E_{2}(\beta+1, k-1, j)-E_{2}(\beta-1, k, j)
$$

and

$$
E_{2}(\alpha, k, j)=\frac{2 \sqrt{P(j) P(k)}(-1)^{k+j}}{\sqrt{\pi} \sqrt{k ! j !}} \sum_{m, p=1}^{k, j} \frac{(-1)^{m+p} \Gamma(((3-\alpha) / 2)+m+p)\left(\begin{array}{c}
k \\
m
\end{array}\right)\left(\begin{array}{l}
j \\
p
\end{array}\right)}{m ! p ! P(m) P(p)} .
$$

Proof. Let

$$
S_{n}(k)=c_{2 k+1}^{-1}(2 k+1) ! \frac{(-1)^{k-n} 2^{2 n+1}}{(k-n) !(2 n+1) !} .
$$

Then

$$
c_{2 k+1}^{-1} h_{2 k+1}(r)=\sum_{n=0}^{k} S_{n}(k) r^{2 n+1}
$$

and

$$
E_{2}(\alpha, k, j)=\sum_{m, p=1}^{k, j} S_{m}(k) S_{p}(j) K(\alpha, m, p)
$$

where

$$
K(\alpha, m, p)=\int_{0}^{\infty} \frac{1}{r^{\alpha}} r^{2 m+1} r^{2 p+1} e^{-r^{2}} d r=\frac{1}{2} \Gamma\left(\frac{3-\alpha}{2}+m+p\right) .
$$

On the other hand, the expression for $E_{1}$ follows from applying (3.10) and (3.11).

If $E_{3}$ and $E_{4}$ are as in the following lemma and $\phi_{\mathrm{el}}(r)=1 /\left(1+r^{2}\right)$, then

$$
\begin{aligned}
F_{1}(k, j)= & E_{3}(2 k+1,2 j+1), \\
F_{2}(k, j)=\frac{1}{2} & \left(\sqrt{\frac{2 k+1}{2}} E_{4}(2 k, 2 j+1)+\sqrt{\frac{2 j+1}{2}} E_{4}(2 k+1,2 j)\right. \\
& \left.\quad-\sqrt{k+1} E_{4}(2 k+2,2 j+1)-\sqrt{j+1} E_{4}(2 k+1,2 j+2)+E_{3}(2 k+1,2 j+1)\right),
\end{aligned}
$$


$F_{3}(k, j)=T_{3}(k, j)-\sqrt{\frac{2 k+1}{2}} E_{3}(2 k, 2 j+1)-\sqrt{k+1} E_{3}(2 k+2,2 j+1)$,
$F_{4}(k, j)=\sqrt{\frac{2 k+1}{2}} E_{3}(2 k, 2 j+1)-\sqrt{k+1} E_{3}(2 k+2,2 j+1)$.

Lemma A.4. For $m, n \in \mathbb{N} \cup\{0\}$, let $I(m, n)$ be as in Lemma A.1:

$$
\begin{aligned}
& E_{3}(m, n)=\frac{1}{c_{m} c_{n}} \int_{0}^{\infty} \frac{1}{1+r^{2}} h_{m}(r) h_{n}(r) e^{-r^{2}} d r \\
& E_{4}(m, n)=\frac{1}{c_{m} c_{n}} \int_{0}^{\infty} \frac{r}{1+r^{2}} h_{m}(r) h_{n}(r) e^{-r^{2}} d r .
\end{aligned}
$$

Then

$$
\begin{aligned}
& E_{3}(0,0)=2 e \int_{0}^{1} e^{-t^{2}} d t+e \sqrt{\pi}, \\
& E_{4}(0,0)=e \sqrt{\pi} \int_{1}^{\infty} \frac{e^{-t}}{t} d t, \\
& E_{3}(m+1,0)=\frac{1}{\sqrt{m+1}}\left(E_{4}(m, 0)-\sqrt{m} E_{3}(m-1,0)\right), \\
& E_{4}(m+1,0)=\frac{1}{\sqrt{m+1}}\left(\sqrt{2} I(m, 0)-\sqrt{2} E_{3}(m, 0)-\sqrt{m} E_{4}(m-1,0)\right), \\
& E_{3}(m+1, n+1)=\frac{1}{\sqrt{(m+1)(n+1)}}\left(2 I(m, n)-2 E_{3}(m, n)+-\sqrt{2 m} E_{4}(m-1, n)\right. \\
& E_{4}(m+1, n+1)=\frac{\left.-\sqrt{2 n} E_{4}(m, n-1)+\sqrt{m n} E_{3}(m-1, n-1)\right),}{\sqrt{(m+1)(n+1)}}\left(\sqrt{2(n+1)} I(m, n+1)-2 E_{4}(m, n)\right. \\
& \left.\quad+\sqrt{2 n} E_{3}(m, n-1)-\sqrt{2 m} E_{4}(m-1, n)+\sqrt{m n} E_{3}(m-1, n-1)\right) .
\end{aligned}
$$

Proof. The recursions for $E_{3}$ and $E_{4}$ follow from (3.11).

Acknowledgements. The authors would like to thank Jean Dolbeault, Michael Levitin, Mathieu Lewin, Éric Séré and Michael Strauss for fruitful discussions during the preparation of this work.

The first author is grateful for the hospitality of CEREMADE and Université de FrancheComté. The second author has been partially supported by ESPRC grant EP/D054621.

\section{References}

1. A. Berthier and V. Georgescu, 'On the point spectrum of Dirac operators', J. Funct. Anal. 71 (1987) 309-338.

2. T. Betcke, N. Higham, V. Mehrmann, C. Schröder and F. Tisseur, NLEVP: A collection of nonlinear eigenvalue problems. http://www.mims.manchester.ac.uk/research/numerical-analysis/nlevp.html.

3. T. Betcke, N. Higham, V. Mehrmann, C. Schröder and F. Tisseur, NlevP: A collection of nonlinear eigenvalue problems. MIMS EPrint 2008.40, Manchester Institute for Mathematical Sciences, The University of Manchester, UK, (April 2008).

4. M. Birman and A. Laptev, 'Discrete spectrum of the perturbed Dirac operator', Ark. Mat. 32 (1994) $13-32$.

5. L. Boulton, 'Limiting set of second order spectra', Math. Comput. 75 (2006) 1367-1382.

6. L. Boulton, 'Non-variational approximation of discrete eigenvalues of self-adjoint operators', IMA $J$. Numer. Anal. 27 (2007) 102-121.

7. L. Boulton and M. Levitin, 'On approximation of the eigenvalues of perturbed periodic Schrodinger operators', J. Phys. A: Math. Theor. 40 (2007) 9319-9329. 
8. K. Dyall, 'Kinetic balance and variational bounds failure in the solution of the Dirac equation in a finite gaussian basis set', Chem. Phys. Lett. 174 (1990) 25-32.

9. M. Esteban, M. Lewin and E. SÉré, 'Variational methods in relativistic quantum mechanics', Bull. AMS 45 (2008) 535-593.

10. M. Griesemer and J. Lutgen, 'Accumulation of discrete eigenvalues of the radial Dirac operator', J. Funct. Anal. 162 (1999) 120-134.

11. P. D. Hislop, 'Exponential decay of two-body eigenfunctions: a review', Proceedings of the Symposium on Mathematical Physics and Quantum Field Theory (Berkeley, CA, 1999), Electron. J. Differ. Equ. Conf., vol. 4 (Southwest Texas State University, San Marcos, TX, 2000) 265-288 (electronic).

12. M. Klaus, 'On the point spectrum of Dirac operators', Helv. Phys. Acta. 53 (1980) 463-482.

13. H. LAnger, M. LANGer and C. Tretter, 'Variational principles for eigenvalues of block operator matrices', Indiana Univ. Math. J. 51 (2002) 1427-1459.

14. M. Levitin and E. ShargorodSky, 'Spectral pollution and second-order relative spectra for self-adjoint operators', IMA J. Numer. Anal. 24 (2004) 393-416.

15. K. M. Schmidt, 'Eigenvalue asymptotics of perturbed periodic Dirac systems in the slow-decay limit', Proc. Amer. Math. Soc. 131 (2003) 1205-1214 (electronic).

16. E. Shargorodsky, 'Geometry of higher order relative spectra and projection methods', J. Operator Theory 44 (2000) 43-62.

17. R. Stanton and S. Havriliak, 'Kinetic balance a partial solution to the problem of variational safety in dirac calculations', J. Chem. Phys. 81 (1984) 1910-1918.

18. M. STRAUSS, 'Quadratic projection methods for approximating the spectrum of self-adjoint operators', IMA J. Numer. Anal., to appear (2010).

19. B. Thaller, The Dirac Equation (Springer, Berlin, 1992).

20. H. Triebel, 'Hardy inequalities in function spaces', Math. Bohem. 124 (1999) 123-130.

\section{Lyonell Boulton}

Ceremade (UMR CNRS 7534)

Université Paris-Dauphine

Place de Lattre de Tassigny, F-75775

Paris Cedex 16

France

and

Department of Mathematics and

Maxwell Institute for Mathematical

Sciences

Heriot-Watt University

Edinburgh, EH14 4AS

United Kingdom

L.Boulton@hw.ac.uk

\author{
Nabile Boussaid \\ Département de Mathématiques \\ UFR Sciences et techniques \\ 16 route de Gray - 25 030, Besançon \\ Cedex \\ France
}

nabile.boussaid@univ-fcomte.fr 